\title{
CURRENTS, SYSTOLES, AND COMPACTIFICATIONS OF CHARACTER VARIETIES
}

\author{
M. BURGER, A. IOZZI, A. PARREAU, AND M. B. POZZETTI
}

\begin{abstract}
Aвstract. We study the Weyl chamber length compactification both of the Hitchin and of the maximal character varieties and determine therein an open set of discontinuity for the action of the mapping class group. This result is obtained as consequence of a canonical decomposition of a geodesic current on a surface of finite type arising from a topological decomposition of the surface along special geodesics. We show that each component either is associated to a measured lamination or has positive systole. For a current with positive systole, we show that the intersection function on the set of closed curves is bilipschitz equivalent to the length function with respect to a hyperbolic metric.
\end{abstract}

\section{Contents}

1. Introduction

1.1. Decomposition of currents

1.2. Systole of a current

1.3. Positive systole and a domain of discontinuity in the Weyl chamber length boundary

1.4. Actions on buildings

1.5. Outline of the paper

Date: December 6, 2021.

2010 Mathematics Subject Classification. 32G15, 22E40.

Marc Burger thanks Francis Bonahon and Kasra Rafi for enlightening conversations on geodesic currents and Kasra Rafi for suggesting to prove a decomposition theorem for geodesic currents. Beatrice Pozzetti thanks Anna Wienhard and Darryl Cooper for insightful conversations.

Beatrice Pozzetti was partially supported by SNF grant P2EZP2_159117, and by DFG project PO 2181/1. Marc Burger and Alessandra Iozzi were partially supported by SNF grant 2-77196-16. Alessandra Iozzi acknowledges moreover support from U.S. National Science Foundation grants DMS 1107452, 1107263, 1107367 "RNMS: Geometric Structures and Representation Varieties" (the GEAR Network). Anne Parreau thanks the Forschungsinstitut für Mathematik for their hospitality. Marc Burger thanks the Leverhulme Trust for supporting his visit to the University of Cambridge as Leverhulme Visiting Professor. Marc Burger, Alessandra Iozzi and Beatrice Pozzetti thank the Isaac Newton Institute for Mathematical Sciences, Cambridge, for support and hospitality during the program "Non-Positive Curvature Group Actions and Cohomology" where work on this paper was undertaken. This work was partially supported by EPSRC grant no $\mathrm{P} / \mathrm{K} 032208 / 1$. 
2. Preliminaries on currents 12

3. Decompositions 15

3.1. The lamination associated to a subset of geodesics in $\mathbb{H} \quad 15$

3.2. The structure of subsets of geodesics in $\Sigma \quad 17$

3.3. Proof of Theorem 1.2 20

4. Straight pseudo-distance, length shortening and systole 20

5. Currents with positive systole 26

6. Currents with vanishing systoles and laminations

7. On the Weyl chamber length compactification

References 37

\section{INTRODUCTION}

Let $S=\Gamma \backslash \mathbb{H}$ be a geometrically finite surface, where $\mathbb{H}$ is the hyperbolic plane and $\Gamma<\operatorname{PSL}(2, \mathbb{R})$ is a finitely generated torsion-free discrete group. A geodesic current on $S$ is a $\Gamma$-invariant Radon measure on the space $\mathcal{G}(\mathbb{H})$ of unoriented, unparametrized geodesics. Geodesic currents occur in many different contexts. For instance they play a fundamental role in the study of hyperbolic structures [Bon01], of negatively curved metrics [Ota90], or of singular flat structures [DLR10]. A crucial fact is that length functions of these structures are intersection functions of geodesic currents. This has been recently extended to Hitchin and maximal representations by Martone and Zhang [MZ19] when $S$ is compact (see also [BCLS18]).

Let $\Sigma \subset S$ be the convex core of $S$. Our main object of study are geodesic currents on $S$ whose support is contained in the subset $\widetilde{\mathcal{G}}(\Sigma)$ of geodesics whose projection is in $\Sigma$. We refer to these as geodesic currents on $\Sigma$ and we denote them by $\mathcal{C}(\Sigma)$. The aim of this paper is to establish structural properties of geodesic currents on $\Sigma$ in terms of their intersection with closed geodesics, and in particular in terms of their systole. Our motivation comes from the study of compactifications of maximal and Hitchin character varieties: as an application, in the case in which $S$ is compact, we construct a natural open domain of discontinuity for the action of the mapping class group of $S$ on the Weyl chamber length boundary of these components and show that in the higher rank case this set is not empty (see $\S$ 1.3). We will also give explicit examples of actions of $\Gamma$ on $\widetilde{A}_{2^{-}}$ buildings whose orbit maps are quasi-isometric embeddings and whose length functions are in this set of discontinuity (see $\S 1.4$ ).

The degree of generality adopted here in our treatment of currents, in particular allowing $S$ to have cusps, turns out to be needed in order to understand all the possible degenerations of maximal or Hitchin representations in higher rank groups. If $\mathbb{F}$ is a non-Archimedean real closed field, the study of maximally framed representations of surface groups 
into $\operatorname{Sp}(2 \mathrm{~m}, \mathbb{F})$ was initiated in [BP17]. In a forthcoming paper we will show how to associate to such a representation $\rho$ a geodesic current $\mu_{\rho}$ whose intersection function on closed geodesics gives the length function, [BIPP21]. Together with the results of this current paper, the assignment $\rho \mapsto \mu_{\rho}$ is a key tool in the study of the "real spectrum" compactification of maximal and Hitchin character varieties and of its nice algebraic geometric properties (see [BIPP20] for an announcement of the results).

1.1. Decomposition of currents. Given a geodesic current $\mu$ on $\Sigma$, we exhibit two laminations with corresponding decompositions of the current and show that the complementary regions are filled by the support of $\mu$ in a specific manner.

We say that a geodesic $g$ in $\Sigma$ is $\mu$-short if no lift thereof intersects transversally a geodesic in the support of $\mu$. The terminology reflects the fact that, in an appropriate sense, the topological intersection of two geodesics generalizes to a concept of length. Note that geodesic currents associated to points in the Hitchin and maximal character varieties of a compact surface $S$ are binding, that is, they have no $\mu$-short geodesics. This is no longer the case for currents associated to points in the boundary of such character varieties and the study of $\mu$-short geodesics will enable us to analyze the structure of such currents.

Given a geodesic lamination $\Lambda \subset \Sigma$ consisting of $\mu$-short geodesics, the current decomposes as a finite sum

$$
\mu=\mu_{\Lambda}+\sum_{\mathcal{R}} \mu_{\mathcal{R}}
$$

that is orthogonal for the Bonahon-intersection form $i(\cdot, \cdot)$ (see $\S 2$ for the definition), where the sum is taken over the complementary regions of $\Lambda$, and $\mu_{\mathcal{R}}$ (respectively $\mu_{\Lambda}$ ) denote the currents on $\Sigma$ given by the restriction of $\mu$ to the set of geodesics projecting into $\mathcal{R}$ (respectively $\Lambda$ ).

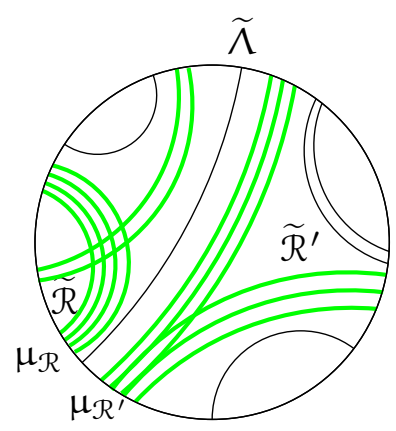

FIgURE 1. The lift $\widetilde{\Lambda}$ to $\mathbb{H}$ of the lamination $\Lambda$, the lifts $\widetilde{\mathcal{R}}$ and $\widetilde{\mathcal{R}^{\prime}}$ of the regions $\mathcal{R}$ and $\mathcal{R}^{\prime}$ and some geodesics in the support of $\mu_{\mathcal{R}}$ and $\mu_{\mathcal{R}^{\prime}}$ 
We consider the set $\Lambda_{\mu}$ of solitary $\mu$-short geodesics, namely $\mu$-short geodesics that don't intersect any other $\mu$-short geodesic

$$
\Lambda_{\mu}:=\left\{c \in \mathcal{G}(\Sigma): \mu \text {-short, and } i\left(c, c^{\prime}\right)=0 \forall c^{\prime} \mu \text {-short }\right\}
$$

and the set $\varepsilon_{\mu}$ of closed $\mu$-short solitary geodesics:

$\mathcal{E}_{\mu}:=\left\{c \in \mathcal{G}(\Sigma)\right.$ : closed, $\mu$-short, and $i\left(c, c^{\prime}\right)=0 \forall c^{\prime}$ closed and $\mu$-short $\}$.

Then $\mathcal{E}_{\mu}$ is a finite collection of pairwise disjoint simple closed geodesics containing the boundary components of $\Sigma$. In particular $\mathcal{E}_{\mu}$ is a geodesic lamination, and $\Lambda_{\mu}^{\mathrm{ref}}=\varepsilon_{\mu} \cup \Lambda_{\mu}$ is a geodesic lamination refining $\varepsilon_{\mu}$.

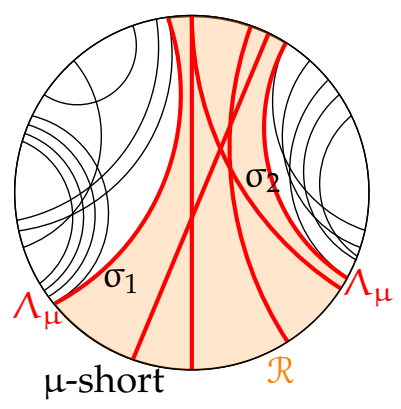

FIGURE 2. All the thick red geodesics in $\widetilde{\mathcal{R}}$ project to $\mu$-short; only $\sigma_{1}$ and $\sigma_{2}$ are in $\widetilde{\Lambda}_{\mu}$.

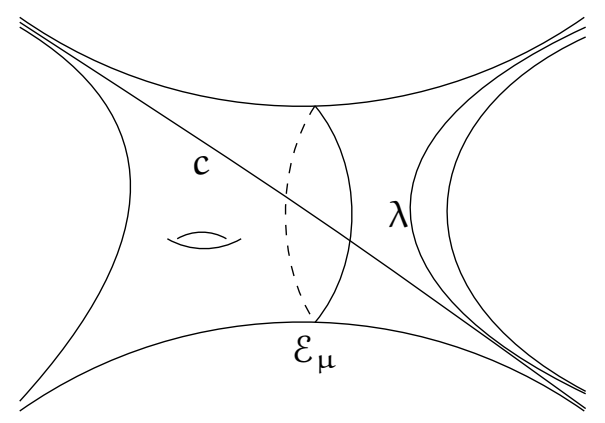

Figure 3. Here $\Lambda=\{\lambda\}$ and $\mu=\delta_{\lambda}$. In this case there is a simple $\mu$-short geodesic $c$ that crosses $\varepsilon_{\mu}$ but it is of course not closed.

Example 1.1 (See Figure 3). Let $S=\Sigma$ be a hyperbolic surface of finite area with at least one cusp, and let $\Lambda \subset S$ be a finite lamination consisting of geodesics with all their endpoints in cusps. Let $\mu=\sum_{c \in \Lambda} \delta_{c}$ be the geodesic current that corresponds to the measured lamination with a Dirac 
mass $\delta_{c}$ along every leaf $c$ of $\Lambda$. In this case $\mathcal{E}_{\mu}$ is the set of boundary components of the smallest subsurface with geodesic boundary containing $\Lambda$ and $\Lambda_{\mu}=\Lambda$.

Theorem 1.2. Let $\mu$ be a geodesic current on the convex core $\Sigma$ of a geometrically finite hyperbolic surface.

(1) Let $\Lambda$ be the lamination $\Lambda=\mathcal{E}_{\mu}$ and let us consider the corresponding decomposition (1.1) of $\mu$. For every complementary region $\mathcal{R}$ of $\mathcal{E}_{\mu}$, either $\mu_{\mathcal{R}}=0$ or every lift of every closed geodesic $\mathrm{c}$ in $\mathcal{R}$ intersects transversally the support $\operatorname{supp}(\mu)$ of $\mu$.

(2) Let $\Lambda$ be the lamination $\Lambda=\Lambda_{\mu}^{\text {ref }}$ and let us consider the corresponding decomposition (1.1) of $\mu$. For every complementary region $\mathcal{R}$ of $\Lambda_{\mu}^{\text {ref }}$, either $\mu_{\mathcal{R}}=0$ or every lift of every geodesic $\mathrm{c}$ in $\mathcal{R}$ intersects transversally $\operatorname{supp}(\mu)$.

If $S=\Sigma$ is compact, part (1) of Theorem 1.2 has been established independently by Erlandsson and Mondello [EM, § 1.5].

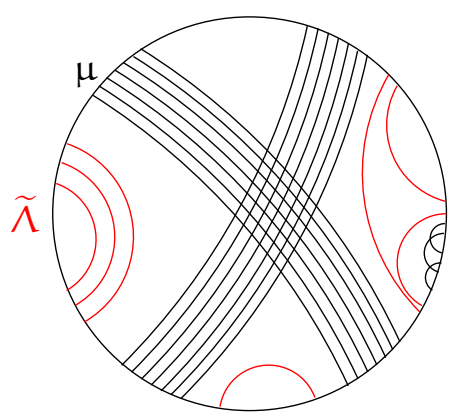

FIgURE 4. The lift of the lamination $\Lambda$ in Theorem 1.2 (2), and some geodesics in the support of $\mu$ (in black).

1.2. Systole of a current. In the first decomposition in Theorem 1.2 let $\Sigma=\Gamma \backslash \mathrm{C}$, where $\mathrm{C} \subset \mathbb{H}$ is the convex hull of the limit set of $\Gamma$ and let $\mathcal{R}$ be a complementary region of $\mathcal{E}_{\mu}$; its metric completion $\Sigma^{\prime}$ is then the quotient $\Gamma^{\prime} \backslash C^{\prime}$ of a closed convex subset $C^{\prime} \subset C$ by a finitely generated subgroup $\Gamma^{\prime}<\Gamma$. We can then restrict $\mu$ to the set of geodesics contained in $C^{\prime}$ and obtain a current $\mu^{\prime}$ on $\Sigma^{\prime}$ such that $i(\mu, c)=i\left(\mu^{\prime}, c\right)$ for every closed geodesic c contained in $\Sigma^{\prime}$. Thus, because of Theorem 1.2(1), in order to study currents on a surface with boundary it is enough to consider currents $\mu$ for which $i(\mu, c)>0$ for every closed geodesic c contained in the interior of the surface.

For a current $\mu$ on $\Sigma$, define then its systole

$$
\operatorname{Syst}(\mu):=\inf i(\mu, c)
$$


where the infimum is taken over all closed geodesics c contained in the interior $\Sigma^{\circ}$ of $\Sigma$. Our next results analyze the structure of $\mu$ depending on the vanishing or the non-vanishing of $\operatorname{Syst}(\mu)$. These results are stated in terms of the intersection properties of $\mu$ with geodesic currents with compact carrier and with compactly supported measured laminations. The carrier $\operatorname{Carr}(\mu) \subset \Sigma$ of a current $\mu$ on $\Sigma$ is the closed subset obtained by projecting to $\Sigma$ all the points lying on the geodesics in the support of $\mu$. For a compact subset $\mathrm{K} \subset \Sigma$, let

$$
\mathcal{C}_{\mathrm{K}}(\Sigma):=\{\mu \in \mathcal{C}(\Sigma): \operatorname{Carr}(\mu) \subset \mathrm{K}\}
$$

and let

$$
\mathcal{M} \mathcal{L}_{\mathfrak{c}}(\stackrel{\circ}{\Sigma})^{\circ}:=\left\{\mathrm{c} \in \mathcal{C}(\Sigma): \mathfrak{i}(\mu, \mu)=0 \text { and } \operatorname{Carr}(\mu) \subset \Sigma^{\circ} \text { is compact }\right\} .
$$

Observe that the condition $i(\mu, \mu)=0$ means that any $\mu \in \mathcal{M} \mathcal{L}_{c}\left(\Sigma^{\circ}\right)$ corresponds to a measured geodesic lamination.

It is easy to see that there is a compact set $K \subset \Sigma^{\circ}$ such that any simple geodesic with compact closure in $\Sigma^{\circ}$ is contained in $K$, so that $\mathcal{M} \mathcal{L}_{\mathcal{c}}\left(\Sigma^{\circ}\right) \subset$ $\mathcal{C}_{\mathrm{K}}(\Sigma)$. Thus we have the following:

Theorem 1.3. Assume that $\Sigma^{\circ}$ is not the thrice punctured sphere. Let $\mathrm{K} \subset \Sigma^{\circ}$ be a compact subset such that $\mathcal{N} \mathcal{L}_{\mathrm{c}}(\stackrel{\circ}{\Sigma}) \subset \mathcal{C}_{\mathrm{K}}(\Sigma)$. Let $\mu$ be a geodesic current on $\Sigma$. Then the following are equivalent:

(1) $\operatorname{Syst}(\mu)>0$;

(2) the function $\lambda \mapsto i(\mu, \lambda)$ does not vanish on $\mathcal{M} \mathcal{L}_{\mathrm{c}}(\stackrel{\circ}{\Sigma}) \backslash\{0\}$;

(3) the function $\lambda \mapsto i(\mu, \lambda)$ does not vanish on $\mathcal{C}_{K}(\Sigma) \backslash\{0\}$;

(4) Every geodesic recurrent in $\Sigma^{\circ}$ intersects transversely some geodesic in the support of $\mu$.

We say that a geodesic $g \in \mathcal{G}(\mathbb{H})$ is recurrent in $\Sigma^{\circ}$ if the projection map from $g$ into $\Sigma^{\circ}$ is not proper. If $\Sigma$ has no cusps, then property (4) in Theorem 1.3 is equivalent to the current $\mu$ being binding in the sense of [EM, Definition 3.1]. If $\Sigma$ is compact and $\partial \Sigma=\varnothing$, every geodesic is recurrent.

Example 1.4. (1) Unlike the case where $\Sigma$ is closed, in general there are geodesic currents with positive systole and whose support is a lamination: for example if in Example 1.1 we take the lamination $\wedge$ such that the complementary regions are ideal polygons, the corresponding current $\mu$ will have positive systole.

(2) The Patterson-Sullivan current (see Example 2.1 (3)) has positive systole.

For the next result we endow the space $\mathcal{C}(S)$ of geodesic currents on $S$, and hence $C(\Sigma)$, with the weak ${ }^{*}$-topology coming from the dual of the topological vector space of continuous compactly supported functions on $\mathcal{G}(\mathbb{H})$. The space $\mathbb{P C}(\Sigma):=\mathbb{R}_{>0} \backslash(\mathcal{C}(\Sigma) \backslash\{0\})$ of projectivized geodesic 
currents is then endowed with the quotient topology and, as such, it is compact metrizable (see Proposition 2.6).

Corollary 1.5. (1) The systole function Syst: $\mathrm{C}(\Sigma) \rightarrow[0, \infty)$ is continuous.

(2) For every current $\mu$ on $\Sigma$, with $\operatorname{Syst}(\mu)>0$ and every compact subset $\mathrm{K} \subset \Sigma^{\circ}$, there are constants $0<\mathrm{c}_{1} \leqslant \mathrm{c}_{2}<+\infty$ such that

$$
c_{1} \ell_{\text {hyp }}(c) \leqslant i(\mu, c) \leqslant c_{2} \ell_{\text {hyp }}(c)
$$

for every closed geodesic $\mathrm{c} \subset \mathrm{K}$. Here $\mathrm{l}_{\mathrm{hyp}}$ denotes the hyperbolic length.

(3) The set $\Omega=\{[\mu] \in \mathbb{P C}(\Sigma)$ : Syst $(\mu)>0\}$ is open in $\mathbb{P C}(\Sigma)$ and the mapping class group of $\Sigma$ acts properly discontinuously on it.

Remark 1.6. If $S=\Sigma$ is a compact surface, in [MZ19] the authors establish interesting systolic inequalities for period minimizing currents with full support, that is, currents with full support such that

$$
\{c \subset S: \text { closed geodesic with } i(\mu, c) \leqslant T\}
$$

is finite for every $T$. In fact our results imply that the first condition is redundant, that is, every current with full support is period minimizing. Indeed by Theorem 1.3 a current with full support has necessarily positive systole. By Corollary 1.5(3) with $K=\Sigma$ this implies the finiteness of the set in (1.2).

Assume now that $\mu$ is a geodesic current on $\Sigma$ such that $i(\mu, c)>0$ for all closed geodesics $c \subset \Sigma^{\circ}$. Then $\varepsilon_{\mu}$ is the set of boundary components of $\Sigma$ and $\Sigma^{\circ}$ is the unique complementary region. According to (1.1) we have a decomposition

$$
\mu=\mu_{\varepsilon_{\mu}}+\mu_{\Sigma}^{\circ}
$$

and the next result gives the structure of the geodesic current $\mu_{\Sigma}$ if the systole of $\mu$ vanishes.

Theorem 1.7. Let $\mu$ be a geodesic current on $\Sigma$ with positive intersection with every closed geodesic $\mathrm{c} \subset \sum^{\circ}$. Then the following are equivalent:

(1) $\operatorname{Syst}(\mu)=0$;

(2) $\mu_{\Sigma}^{\circ}$ corresponds to a measured lamination, compactly supported in $\Sigma^{\circ}$, minimal and surface filling.

We say that a geodesic lamination is surface filling if every complementary region is either an ideal polygon, or an ideal polygon bounding either a boundary geodesic or a cusp.

Remark 1.8. The relation between our results and the ones in [EM] is the following. If $\Sigma$ is compact, it follows from Theorem 1.2(1) that a current $\mu$ of full hull in the sense of [EM] is one such that $i(\mu, c)>0$ for every closed geodesic $c \subset \sum^{\circ}$. Then Theorem 1.3 and Theorem 1.7 show that the dichotomy "Syst $(\mu)>0$ " or "Syst $(\mu)=0$ " gives for $\mu$ of full hull the dichotomy " $\mu$ is binding" or " $\mu$ is a measured lamination". That this is a dichotomy for currents of full hull is the content of [EM, Theorem 3.19]. 
In general we consider the decompostion

$$
\Sigma=\bigcup_{v \in \mathcal{V}_{\mu}} \Sigma_{v}
$$

as a union of subsurfaces with geodesic boundary induced by the set $\varepsilon_{\mu}$ closed $\mu$-short solitary geodesics. For any such a subsurface $\Sigma_{v}$ and for a geodesic current $\tau$ let

$$
\text { Syst }_{\Sigma_{v}}(\tau)=\inf \left\{\dot{i}(\tau, c) \mid c \subset \Sigma_{v}^{\circ} \text { closed geodesic }\right\} .
$$

Combining Theorem 1.3 and Theorem 1.7 we deduce

Corollary 1.9. Let $\mu$ be a geodesic current on a complete hyperbolic surface of finite area $\Sigma=\Gamma \backslash \mathrm{H}^{2}$, and let $\mathcal{V}_{\mu}$ be as in (1.3). We have

$$
\mu=\sum_{v \in \mathcal{V}_{\mu}} \mu_{\Sigma_{v}}+\sum_{c \in \varepsilon_{\mu}} \lambda_{c} \delta_{c}
$$

where $\delta_{\mathrm{c}}$ is the geodesic current associated to the closed geodesic c. Furthermore, for every $\nu \in \mathcal{V}_{\mu}$ for which $\mu_{\Sigma_{v}} \neq 0$ precisely one of the following holds:

(1) either Syst $_{\Sigma_{v}}\left(\mu_{v}\right)>0$,

(2) or $\mu_{v}$ is the geodesic current associated to a measured lamination compactly supported in $\Sigma_{v}^{\circ}$ and surface filling in $\Sigma_{v}$.

1.3. Positive systole and a domain of discontinuity in the Weyl chamber length boundary. Let $\Gamma<\operatorname{PSL}(2, \mathbb{R})$ be a (not necessarily torsion-free) cocompact lattice and let $G$ be either $\operatorname{PSL}(n, \mathbb{R})$ or $\operatorname{Sp}(2 m, \mathbb{R})$. Let $X(\Gamma, G)$ be the space of G-conjugacy classes of representations of $\Gamma$ in $G$ that are Hitchin if $G=\operatorname{PSL}(n, \mathbb{R})$ or maximal if $G=\operatorname{Sp}(2 m, \mathbb{R})$ (see $\S \mathbb{7}$ ). Our objective is to apply our results on currents to the study of the action of the mapping class group on the Weyl chamber length boundary $\partial \chi(\Gamma, \mathrm{G})$. Recall (see [Par12]) that $\partial X(\Gamma, \mathrm{G})$ is a compact subset of the space $\mathbb{P}\left(\mathfrak{C}^{\Gamma}\right)$ of projective classes of functions from $\Gamma$ to a closed Weyl chamber $\mathfrak{C}$ of $G$, and that a diverging sequence $\left(\left[\rho_{k}\right]\right)_{k}$ in $X(\Gamma, G)$ converges to the projective class [L] of a nonzero function $L: \Gamma \rightarrow \mathfrak{C}$ if and only if $\left[\lambda \circ \rho_{k}\right]$ converges to [L] in $\mathbb{P}\left(\mathfrak{C}^{\Gamma}\right)$, where $\lambda: G \rightarrow \mathfrak{C}$ is the Jordan projection, see (7.2). Let $\|\cdot\|$ denote a Weyl group invariant norm on the Cartan subalgebra $\mathfrak{a} \supset \mathfrak{C}$ and define the systole of a function $L: \Gamma \rightarrow \mathfrak{C}$ by

$$
\operatorname{Syst}(\mathrm{L}):=\inf _{\substack{\gamma \in \Gamma \\ \gamma \text { hyperbolic }}}\|\mathrm{L}(\gamma)\| .
$$

Observe that the positive systole subset

$$
\Omega(\Gamma, \mathrm{G}):=\{[\mathrm{L}] \in \partial \mathcal{X}(\Gamma, \mathrm{G}): \operatorname{Syst}(\mathrm{L})>0\}
$$

of $\partial X(\Gamma, G)$ is well-defined and independent of the choice of the norm $\|\cdot\|$.

The next result is a consequence of Corollary 1.5 and [MZ19, Theorem 1.1]: 
Corollary 1.10. Let $\Gamma<\operatorname{PSL}(2, \mathbb{R})$ be a cocompact lattice and let $X(\Gamma, \mathrm{G})$ be the character variety of representations of $\Gamma$ in $\mathrm{G}$ that are either Hitchin or maximal.

(1) $\Omega(\Gamma, G)$ is an open subset of $\partial X(\Gamma, G)$.

(2) For every $[\mathrm{L}] \in \Omega(\Gamma, \mathrm{G})$ there are constants $0<\mathrm{c}_{1} \leqslant \mathrm{c}_{2}<\infty$ such that, for every hyperbolic element $\gamma \in \Gamma$,

$$
c_{1} \ell_{\text {hyp }}(\gamma) \leqslant\|L(\gamma)\| \leqslant c_{2} \ell_{\text {hyp }}(\gamma),
$$

where $\ell_{\text {hyp }}(\gamma)$ is the translation length of $\gamma$ in $\mathbb{H}$.

(3) Assume that $\Gamma$ is torsion-free. Then the mapping class group $\mathcal{M} C \mathcal{G}(\mathrm{S})$ of $\mathrm{S}:=\Gamma \backslash \mathbb{H}$ acts properly discontinuously on $\Omega(\Gamma, \mathrm{G})$.

Note that if $\mathrm{G}=\operatorname{PSL}(2, \mathbb{R})$ and $\Gamma$ is torsion-free, $\partial X(\Gamma, \mathrm{G})$ is the Thurston boundary of the Teichmüller space of $S=\Gamma \backslash \mathbb{H}$ and it is well-known that $\operatorname{Syst}(\mathrm{L})=0$ for every $[\mathrm{L}] \in \partial X(\Gamma, \mathrm{G})$. It is a striking fact that it is not anymore the case when $\mathrm{G}$ has higher rank.

Corollary 1.11. Let $S=\Gamma \backslash \mathbb{H}$ be any compact hyperbolic surface, and $\mathrm{G}=$ $\operatorname{PSL}(n, \mathbb{R})$ with $n \geqslant 3$ or $\mathrm{G}=\operatorname{Sp}(2 \mathrm{~m}, \mathbb{R})$ with $\mathrm{m} \geqslant 2$. Then the positive systole subset $\Omega(\Gamma, \mathrm{G})$ is non-empty.

It is a natural question whether length functions if $\Omega(\Gamma, \mathrm{G})$ correspond to some kind of geometric structures on the surface $S=\Gamma \backslash \mathbb{H}$. In the case where $G=\operatorname{PSL}(2, \mathbb{R}) \times \operatorname{PSL}(2, \mathbb{R})$, one can show using [GM91] that $\Omega(\Gamma, G)$ corresponds to the Teichmüller space of semi-translation structures $S$. We will study this case in further details in a forthcoming paper.

Another interesting feature of the positive systole subset is its relationship with entropy. This uses [MZ19, Corollary 1.5].

Corollary 1.12. Let $S=\Gamma \backslash \mathbb{H}$ be a compact hyperbolic surface and $G=\operatorname{PSL}(n, \mathbb{R})$ or $\mathrm{G}=\mathrm{Sp}(2 \mathrm{~m}, \mathbb{R})$. Assume that $\left(\left[\rho_{\mathrm{k}}\right]\right)_{\mathrm{k}}$ is a sequence in $X(\Gamma, \mathrm{G})$ converging to a point of $\Omega(\Gamma, \mathrm{G})$. Then we have for the entropy $\mathrm{h}\left(\rho_{\mathrm{k}}\right)$ of $\rho_{\mathrm{k}}$ :

$$
\lim _{k \rightarrow \infty} h\left(\rho_{k}\right)=0 \text {. }
$$

The first examples of such sequences were obtained by $X$. Nie [Nie15] for PSL $(3, \mathbb{R})$ and by T. Zhang in [Zha15a] for PSL $(n, \mathbb{R})$ (see also [Zha15b]). Examples 1.15 and 1.16 below satisfy this property.

Corollary 1.11 is in fact a consequence of next theorem (Theorem 1.13) concerning the Weyl chamber length boundary of the Hitchin component of a hyperbolic triangle group

$$
\Delta:=\Delta(\mathrm{p}, \mathrm{q}, \mathrm{r})=\left\langle\mathrm{a}, \mathrm{b}: \mathrm{a}^{\mathrm{p}}=\mathrm{b}^{\mathrm{q}}=(\mathrm{ab})^{\mathrm{r}}=\mathrm{e}\right\rangle .
$$

Theorem 1.13. Let $\Delta$ be a hyperbolic triangle group and $\mathrm{G}=\operatorname{PSL}(\mathrm{n}, \mathbb{R})$ or $\mathrm{G}=\mathrm{Sp}(2 \mathrm{~m}, \mathbb{R})$. Then for every $[\mathrm{L}] \in \partial X(\Delta, \mathrm{G})$

$$
\text { Syst(L) }>0 \text {. }
$$


1.4. Actions on buildings. Recall that any projectivized function $[\mathrm{L}]$ in $\partial X(\Gamma, G)$ is the $\mathfrak{C}$-valued length function of an action of $\Gamma$ on an affine Bruhat-Tits building, that is typically not simplicial, see [Par12]. In the case where $[\mathrm{L}]$ has positive systole, that is $[\mathrm{L}] \in \Omega(\Gamma, \mathrm{G})$, by Corollary $1.10(2)$ the action of $\Gamma$ is displacing in the sense of [DGLM11], and in particular orbit maps are quasi-isometric embeddings.

Explicit examples of $\Delta$-actions on a simplicial building whose length function belongs to $\partial X(\Delta, \operatorname{SL}(n, \mathbb{R}))$ can be obtained as follows from representations $\rho: \Delta \rightarrow \operatorname{SL}(n, \mathbb{R}(X))$, where $\mathbb{R}(X)$ is the field of rational functions. Let $\mathbb{K}=\mathbb{R}\left[\left[X^{-1}\right]\right]$ be the field of Laurent series endowed with its canonical non-Archimedean $\mathbb{Z}$-valued valuation $v$ for which $v(X)=-1$. Let $B_{n}(\mathbb{K})$ be the Bruhat-Tits building of $\operatorname{SL}(n, \mathbb{K})$. The $\mathfrak{C}$-valued length of $\mathrm{g}$ in $\mathrm{SL}(\mathrm{n}, \mathbb{K})$ is its Jordan projection

$$
\lambda(g)=\left(-v\left(a_{1}\right), \ldots,-v\left(a_{n}\right)\right)
$$

where $a_{1}, \ldots, a_{n}$ are the eigenvalues of $g$ (see $\S 7$ ).

Corollary 1.14. Assume that $\rho: \Delta \rightarrow \mathrm{SL}(\mathrm{n}, \mathbb{R}(X))$ is a representation such that

(i) $\operatorname{tr} \rho\left(\gamma_{0}\right) \in \mathbb{R}(X)$ has a pole at infinity for some $\gamma_{0} \in \Delta$, and

(ii) for all $\mathrm{t} \in \mathbb{R}$ large enough, the specialization $\rho_{\mathrm{t}}$ of $\rho$ at $\mathrm{t}$ is a Hitchin representation.

Then

(1) $[\lambda \circ \rho] \in \Omega(\Delta, \operatorname{SL}(\mathrm{n}, \mathbb{R}))$.

(2) Any $\Delta$-orbit in $\mathrm{B}_{\mathrm{n}}(\mathbb{R}(\mathrm{X}))$ is a quasi-isometric embedding.

We now give explicit examples of representations of triangle groups satisfying the hypotheses of Corollary 1.14.

Example 1.15. For $\Delta=\Delta(3,3,4)$, define

$$
\begin{aligned}
\rho(a) & =\left(\begin{array}{lll}
0 & 0 & 1 \\
1 & 0 & 0 \\
0 & 1 & 0
\end{array}\right) \\
\rho(b) & =\left(\begin{array}{ccc}
1 & 2-X+X^{2} & 3+X^{2} \\
0 & -2+2 X-X^{2} & -1+X-X^{2} \\
0 & 3-3 X+X^{2} & (-1+X)^{2}
\end{array}\right) .
\end{aligned}
$$

According to the main result of [LRT11], $\rho_{t}$ belongs to the SL $(3, \mathbb{R})$-Hitchin component of $\Delta=\Delta(3,3,4)$ for every $t \in \mathbb{R}$. In addition one verifies that

$$
\operatorname{tr}\left(\rho\left(a^{-1} b\right)\right)=2 X^{2}-3 X+6 .
$$

Example 1.16. The following example is due to Goldman, [Gol88, §6]. Let $\Delta=\Delta(p, q, r)$ with $\min (p, q, r) \geqslant 3$. Consider the following matrix with coefficients in $\mathbb{R}(X)$, where $\epsilon_{s}:=\cos \left(\frac{2 \pi}{s}\right)$ for $s>0$,

$$
B(X)=\left(\begin{array}{ccc}
1 & -X^{-1} \epsilon_{p} & -\epsilon_{q} \\
-X \epsilon_{p} & 1 & -\epsilon_{r} \\
-\epsilon_{q} & -\epsilon_{r} & 1
\end{array}\right)
$$


Define $\rho\left(r_{i}\right):=-I d+2 B(X) e_{i}{ }^{t} e_{i}$ where $e_{1}, e_{2}, e_{3}$ are the canonical basis vectors of $\mathbb{R}(X)^{3}$. Then

$$
\begin{aligned}
& \rho(a)=\rho\left(r_{1}\right) \rho\left(r_{2}\right)=\left(\begin{array}{ccc}
-1 & -2 X^{-1} \epsilon_{p} & 0 \\
2 \epsilon_{p} & -1+4 \epsilon_{p}^{2} & 0 \\
2 \epsilon_{\mathrm{q}} & 2 \epsilon_{\mathrm{r}}+4 X^{-1} \epsilon_{\mathrm{p}} \epsilon_{\mathrm{q}} & 1
\end{array}\right) \\
& \rho(b)=\rho\left(r_{2}\right) \rho\left(r_{3}\right)=\left(\begin{array}{ccc}
1 & 2 X^{-1} \epsilon_{\mathrm{p}} & 2 \epsilon_{\mathrm{q}}+4 X^{-1} \epsilon_{\mathrm{p}} \epsilon_{\mathrm{r}} \\
0 & -1 & -2 \epsilon_{\mathrm{r}} \\
0 & 2 \epsilon_{\mathrm{r}} & -1+4 \epsilon_{\mathrm{r}}^{2}
\end{array}\right)
\end{aligned}
$$

define a representation $\rho: \Delta \rightarrow \operatorname{SL}(3, \mathbb{R}(X))$, whose specialization $\rho_{t}$ at all $t>0$ is Hitchin. In addition a computation gives

$$
\operatorname{tr}\left(\rho\left(a^{-1} b\right)\right)=8 \epsilon_{p} \epsilon_{q} \epsilon_{r}\left(X+X^{-1}\right)+16 \epsilon_{p}^{2} \epsilon_{r}^{2}+4 \epsilon_{q}^{2}-1
$$

hence $\rho$ satisfies the hypotheses of Corollary 1.14 provided $p, q, r \neq 4$.

1.5. Outline of the paper. After some preliminaries on currents in $\S 2$, we study in $\S 3$ a general set of geodesics $A$ in $\mathcal{G}(\mathbb{H})$ and associate to it a lamination using the intersection graph of $A$. If $A$ is invariant under $\Gamma$, we deduce, using the structure of complementary regions of laminations in $\Sigma$, general results from which Theorem 1.2 follows.

The main goal of $\S 4$ is to show that the systole of a current can be computed using only simple closed geodesics, provided $\Sigma^{\circ}$ is not the thrice punctured sphere. To this end we associate to any geodesic current on $\mathbb{H}$ a pseudo-distance on $\mathbb{H}$ that is a modification of a pseudo-distance introduced by Glorieux [Glo] and which has the advantage of being additive on colinear triples of points. When $\mu$ is a geodesic current on a hyperbolic surface $S=\Gamma \backslash \mathbb{H}$, this pseudo-distance leads to a pseudo-length for paths and closed curves on $S$ and the main point consists then in showing a Length-Shortening-Under-Surgery Lemma (Lemma 4.9). This is essential in the proof of Theorem 1.3 and Theorem 1.7.

In $\S 5$ we deal with currents of positive systole. The main point in the proof of Theorem 1.3 consists in showing that positive systole currents do not admit $\mu$-short recurrent geodesics. This is shown in Proposition 5.1 using the classical Closing Lemma.

In $\$ 6$, we prove Theorem 1.7, which follows essentially from a study of geodesic laminations consisting of $\mu$-short geodesics.

In $\S 7$ we apply the results on currents to the study of the Weyl chamber length boundary of the Hitchin or maximal components of a cocompact lattice $\Gamma<\operatorname{PSL}(2, \mathbb{R})$. Beside the results of [MZ19], an essential input is Theorem 7.2 establishing that for a hyperbolic triangle group $\Delta$, $\Delta$-invariant non-vanishing currents have positive systole. The basis for the construction of the explicit examples in $\S 1.4$ is Corollary 1.14, which relies on Theorem 7.2 as well as on Puiseux's theorem on the representability of elements of a specific real closure of $\mathbb{R}(X)$ by convergent Puiseux series. 


\section{Preliminaries on CURRents}

A geodesic current is a positive Radon measure on the space $\mathcal{G}(\mathbb{H})$ of unoriented, unparametrized, geodesics in $\mathbb{H}$. The topology on $\mathcal{G}(\mathbb{H})$ is obtained by identifying this space with the quotient by the flip $\sigma(a, b)=$

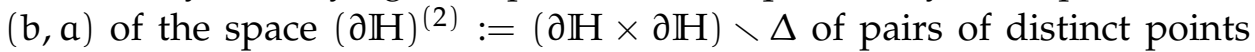
in the boundary $\partial \mathbb{H}$ of the hyperbolic plane $\mathbb{H}$. Via this identification we will think of a geodesic current as a $\sigma$-invariant positive Radon measure on the locally compact space $(\partial \mathbb{H})^{(2)}$.

Let $\Gamma<\operatorname{PSL}(2, \mathbb{R})$ be a torsion-free discrete subgroup and $S=\Gamma \backslash \mathbb{H}$ be the quotient hyperbolic surface. We denote by $\pi: \mathbb{H} \rightarrow S$ the corresponding projection.

A geodesic current on $\mathrm{S}$ is a $\Gamma$-invariant geodesic current.

Examples 2.1. The following examples of geodesic currents will play an important role in the rest of the paper:

(1) The Liouville current $\mathcal{L}$ is the unique (up to positive scaling) $\operatorname{PSL}(2, \mathbb{R})$ invariant Radon measure on $(\partial \mathbb{H})^{(2)}$. It is of course $\Gamma$-invariant for every subgroup $\Gamma<\operatorname{PSL}(2, \mathbb{R})$.

(2) Let $c$ be a geodesic in $S$. Then the set of lifts of $c$ to $\mathbb{H}$ is a $\Gamma$-orbit in $\mathcal{G}(\mathbb{H})$, which is discrete in $\mathcal{G}(\mathbb{H})$ if and only if $c$ is closed as a subset of $S$. Then the sum $\delta_{c}$ of the Dirac masses along this orbit is a geodesic current on $S$.

(3) Let $\mathbb{D}=\{z \in \mathbb{C}:|z|<1\}$ be the unit disk model of $\mathbb{H}$ and $\Gamma<$ $\operatorname{PSU}(1,1)$ be a discrete subgroup. For $\delta \geqslant 0$ a $\delta$-density on $\partial \mathrm{D}$ is a probability measure $v$ such that $v\left(f \circ \gamma^{-1}\right)=v\left(j_{\gamma}^{\delta} \cdot f\right)$ where

$$
j_{\gamma}(\xi)=\frac{1}{|a \xi+b|^{2}}, \quad \gamma=\left(\begin{array}{cc}
\frac{a}{b} & \bar{a}
\end{array}\right) .
$$

For instance the round measure

$$
\lambda(f)=\frac{1}{2 \pi} \int_{0}^{2 \pi} f\left(e^{i \theta}\right) d \theta
$$

is a 1-density for $\operatorname{PSU}(1,1)$. Given a $\delta$-density $v$, the measure $\mu$ on $(\partial \mathbb{D})^{(2)}$ given by

$$
\mu(f)=\iint \frac{f(\xi, \eta)}{|\xi-\eta|^{2 \delta}} d v(\xi) d v(\eta)
$$

is then a $\Gamma$-invariant current.

When $\Gamma$ is finitely generated, and $\delta$ is the critical exponent of $\Gamma$, there is a unique $\delta$-density $\mu$ on $\partial \mathbb{D}$; its support is precisely the limit set $\Lambda$ and the corresponding measure $\mu_{\mathrm{PS}}$ is the PattersonSullivan current. It is thus a current on the convex core $\Sigma$ of $S$ and every recurrent geodesic in $\Sigma^{\circ}$ intersects transversely a geodesic in the support of $\mu_{\mathrm{PS}}$. It follows from Theorem 1.3 that Syst $\left(\mu_{\mathrm{PS}}\right)>0$ and $\mu_{\mathrm{PS}}$ satisfy the conclusion of Corollary 1.5 (2). 
Recall that if $g, h \in \mathcal{G}(\mathbb{H})$ are two geodesics, their intersection number $i(g, h)$ is defined as

$$
i(g, h):= \begin{cases}0 & g, h \text { are disjoint or coincide } \\ 1 & g, h \text { intersect transversally. }\end{cases}
$$

Then the intersection $i(\mu, v)$ of two currents $\mu, v$ on $S$ is defined as follows (see [Bon01] or [Mar]). Let

$$
\mathcal{D G}(\mathbb{H}):=\{(\mathrm{g}, \mathrm{h}) \in \mathcal{G}(\mathbb{H}) \times \mathcal{G}(\mathbb{H}): \mathfrak{i}(\mathrm{g}, \mathrm{h})=1\} ;
$$

then $\operatorname{PSL}(2, \mathbb{R})$ acts properly on the open set $\mathcal{D G}(\mathbb{H})$ and so does $\Gamma$. The intersection $i(\mu, v)$ is then the $(\mu \times v)$-measure of any Borel fundamental domain for the $\Gamma$-action on $\mathcal{D G}(\mathbb{H})$. We will often denote $i\left(\mu, \delta_{c}\right)$ by $i(\mu, c)$.

Examples 2.2. (1) Given two distinct closed geodesics $c, c^{\prime}$ in $S$, the intersection $i\left(\delta_{c}, \delta_{c^{\prime}}\right)$ is the minimal geometric intersection number between two closed curves in the free homotopy classes represented by $c$ and $c^{\prime}$. Instead $i\left(\delta_{c}, \delta_{c}\right)$ is the number of self intersections of the geodesic $c$, so for example $i\left(\delta_{c}, \delta_{c}\right)=0$ if and only if $c$ is simple.

(2) If $\mathrm{c}$ is a closed geodesic in $S$ and $\ell_{\text {hyp }}(\mathrm{c})$ is its hyperbolic length, then we have

$$
i\left(\mathcal{L}, \delta_{c}\right)=\ell_{\text {hyp }}(\mathrm{c}) .
$$

The set $\mathcal{C}(S)$ of geodesic currents on $S$ is a convex cone in the dual of the space of compactly supported functions on $\mathcal{G}(\mathbb{H})$; the latter is provided with the topology of inductive limit of Banach spaces and $\mathcal{C}(S)$ will be equipped with the corresponding weak ${ }^{*}$ topology. Given a geodesic current $\mu$ on $S$, its support $\operatorname{supp}(\mu) \subseteq \mathcal{G}(\mathbb{H})$ is a closed $\Gamma$-invariant subset; as mentioned in the introduction, we call the carrier of $\mu$ and denote by $\operatorname{Carr}(\mu)$ the closed subset of $S$ consisting of the projection to $S$ of the union of all points on all geodesics in $\operatorname{supp}(\mu)$. For $F \subset S$, we denote by $\mathcal{C}_{F}(S)$ the subset of geodesic currents on $S$ with carrier included in $F$.

It is straightforward to verify that if $\mu$ is any current on $S$ and $v$ has compact carrier, then $i(\mu, v)<+\infty$.

Example 2.3. The geodesic current $\delta_{c}$ on $S$ from Example 2.1(2) is a geodesic current on $\Sigma$ if and only if $c$ is either a closed geodesic or an ideal geodesic, that is a geodesic connecting two cusps of $\Sigma$. This is the case in Figure 3 .

One of the most fundamental facts concerning the intersection is the following continuity property due to Bonahon:

Theorem 2.4 ([Bon86, § 4.2]). For every compact subset $\mathrm{K} \subset \mathrm{S}$, the intersection

$$
i: \mathcal{C}(\mathrm{S}) \times \mathcal{C}_{\mathrm{K}}(\mathrm{S}) \rightarrow \mathbb{R}
$$

is continuous. 
This continuity relies on the following crucial technical point that we will use in this paper and that can be found for example in [Mar, Proposition 8.2.8]. It rules out a situation in which a geodesic current may have a "one-sided" atom. Recall that a pencil is a subset of $(\partial \mathbb{H})^{(2)}$ of the form $\{p\} \times B$ where $B \subset \partial \mathbb{H}$ is a Borel subset not containing $p$. The lemma holds as stated for any hyperbolic surface $S=\Gamma \backslash \mathbb{H}$. If $S$ is geometrically finite, then a point in the limit set is conical if and only if it is not a cusp.

Lemma 2.5. Let $\mu$ be a geodesic current on $\mathrm{S}$. Assume that $\mathrm{p} \in \partial \mathrm{H}$ is a conical limit point and the pencil $\{\mathrm{p}\} \times \mathrm{B}$ does not contain the axis of a hyperbolic element. Then

$$
\mu(\{p\} \times B)=0 .
$$

For our purposes we will need the following compactness property, which can be derived from the arguments in [Bon88, Proposition 4].

Proposition 2.6. $\quad$ (1) Let $\mathrm{K} \subset \mathrm{S}$ be compact and let $\mathcal{L}$ be the Liouville current on $\mathrm{S}$. Then the set

$$
\left\{\mu \in \mathcal{C}_{\mathrm{K}}(\mathrm{S}): \mathfrak{i}(\mathcal{L}, \mu)=1\right\}
$$

is compact and hence $\mathbb{P C}_{\mathrm{K}}(\mathrm{S})$ is compact.

(2) The space $\mathbb{P C}(\Sigma)$ is compact.

Proof. The proof of (1) follows the one in [Bon88, Proposition 4] observing that, since $\operatorname{supp}(\mathcal{L})=\mathcal{G}(\mathbb{H})$, any non-zero current $\mu$ on $S$ has positive intersection with $\mathcal{L}$. For the second assertion replace [Bon88, Proposition 4] by the following lemma.

Lemma 2.7. Let $v$ be a current on $\Sigma$ with compact carrier such that every geodesic in $\mathrm{H}$ projecting into $\sum^{\circ}$ intersects transversally a geodesic in the support of $v$. Let $\psi: \mathcal{G}(\mathbb{H}) \rightarrow[0, \infty)$ be continuous with compact support such that for every boundary component $\mathrm{g}$ of $\Sigma, \psi(\tilde{\mathrm{g}})=1$ for some lift $\tilde{\mathrm{g}}$ of $\mathrm{g}$. Then the set

$$
\{\mu \in \mathcal{C}(\Sigma): \mu(\psi)+i(\mu, v) \leqslant 1\}
$$

is compact.

The proof of Lemma2.7is a straightforward modification of the proof of [Bon88, Proposition 4]. The lemma implies Proposition 2.6(2) by observing that the set

$$
P:=\{\mu \in \mathcal{C}(\Sigma): \mu(\psi)+i(\mu, v)=1\}
$$

is compact and the projection map $\mathrm{P} \rightarrow \mathbb{P C}(\Sigma)$ is a continuous bijection.

We refer for instance to [Mar, \$8.3.4] for the notion of measured geodesic lamination on a general hyperbolic surface $S=\Gamma \backslash \mathbb{H}$, and the bijective correspondence between geodesics currents $\mu$ on $S$ with $i(\mu, \mu)=0$, equivalently such that no two geodesics in the support of $\mu$ intersect transversally, and measured geodesic laminations on $S$. 


\section{Decompositions}

Let $S$ be a hyperbolic surface (not necessarily complete) and $F \subset S$ a subset. We denote by $\mathcal{G}(F)$ the set of unoriented, unparametrized geodesics of $S$ that are contained in F. Given a geometrically finite surface $S$ with convex core $\Sigma$ and an arbitrary subset $A \subset \mathcal{G}(\Sigma)$ of geodesics, our aim is to show how one can associate two laminations $\mathcal{E}_{A}$ and $\Lambda_{A}^{\text {ref }}$ respectively, such that their complementary regions are either completely avoided by $A$ or filled by $A$ in two specific ways (see Proposition 3.2 and 3.3). Applying these propositions to the support of a geodesic current on $\Sigma$ will imply Theorem 1.2. In $\S 3.1$ we start by studying the case of a subset of $\mathcal{G}(\mathbb{H})$, then move to geometrically finite surfaces in $\$ 3.2$. where we establish the main propositions. We show in $\$ 3.3$ how to deduce Theorem 1.2 .

3.1. The lamination associated to a subset of geodesics in $\mathbb{H}$. Given a subset $A \subset \mathcal{G}(\mathbb{H})$ its intersection graph, $\operatorname{Graph}(A)$, is the graph whose vertex set is $A$ and two vertices $a, a^{\prime}$ are adjacent if $i\left(a, a^{\prime}\right)=1$. We say that $A$ is $i-$ connected if $\operatorname{Graph}(A)$ is connected; an $i$-connected component $(i-c c) A^{\prime} \subset A$ is then the set of vertices of a connected component of $\operatorname{Graph}(A)$. We proceed to define the lamination associated to $A$, this relies on classical properties of convex hulls in $\mathbb{H}$.

For $A \subset \mathcal{G}(\mathbb{H})$ let Hull $(A) \subset \mathbb{H}$ be the convex hull of the union of the geodesics in $A$, namely the intersection of all convex subsets containing A. Whenever $|A|>1$, then $\Omega:=\operatorname{Hull}(A)$ is in general neither open nor closed but its closure $\bar{\Omega}$ is the closed convex hull of the set $\mathrm{T} \subset \partial \mathrm{H}$ of extremities of geodesics in $A$. Each connected component of $\partial \mathbb{H} \backslash \overline{\mathrm{T}}$ is an interval to which we can associate the geodesic connecting its endpoints; the boundary of $\bar{\Omega}$ in $\mathbb{H}$ is the disjoint union of the set $\Delta(\Omega)$ of all such geodesics.

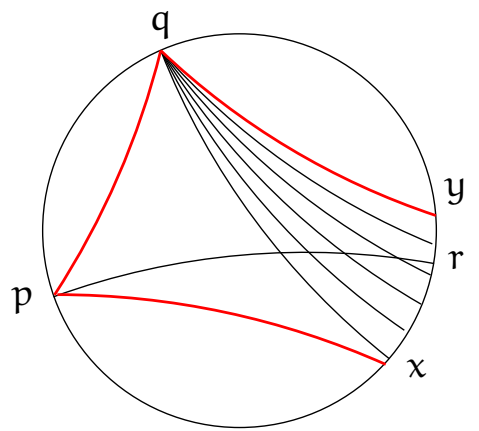

Figure 5. The set $A$ consists of the pencil $\{q\} \times(x, y)$ together with the geodesic $\{p, r\}$. In this case $T$ consists of the union of the open interval $(x, y)$ and the two points $\{p, q\}$. Its boundary has three connected components, and $\Delta(\Omega)$ consists of the three geodesics in red. 
Let

$$
\Lambda_{A}:=\bigcup_{A^{\prime} \text { icc of } A} \Delta\left(\operatorname{Hull}\left(A^{\prime}\right)\right)
$$

be the closure in the space $\mathcal{G}(\mathbb{H})$ of the set of the boundary geodesics of convex hulls of $i$-connected components of $A$. Notice that the boundary of the closure of $\operatorname{Hull}\left(A^{\prime}\right)$ is a lamination for every $i$-cc $A^{\prime}$ and the gist of next proposition is to show that $\Lambda_{A}$ is a lamination as well.

Given any subsets $A, B \subset \mathcal{G}(\mathbb{H})$ we set

$$
i(A, B)=\sum_{g \in A, h \in B} i(g, h) \in[0, \infty] .
$$

Observe that $i(A, B)>0$ if and only if some $a \in A$ intersects transversally some $b \in B$. Then

$$
A^{0}=\{g \in \mathcal{G}(\mathbb{H}): i(g, A)=0\}
$$

is a closed subset of $\mathcal{G}(\mathbb{H})$ and if $A \subset B$, then $B^{0} \subset A^{0}$. Setting $A^{00}:=\left(A^{0}\right)^{0}$, then $A \subset A^{00}$, and $A^{0} \cap A^{00}$ is a lamination, since $i\left(A^{0} \cap A^{00}, A^{0} \cap A^{00}\right) \leqslant$ $i\left(A^{0}, A^{00}\right)=0$.

Lemma 3.1. Let $h$ be a geodesic and $A^{\prime}$ an $i$-cc of $A$. If $i\left(h, \Delta\left(\operatorname{Hull}\left(A^{\prime}\right)\right)>0\right.$, then $i\left(h, A^{\prime}\right)>0$. As a consequence

$$
i\left(\Delta\left(\operatorname{Hull}\left(A^{\prime}\right)\right), \Delta\left(\operatorname{Hull}\left(A^{\prime \prime}\right)\right)\right)=0
$$

for every two $i-c c A^{\prime}, A^{\prime \prime}$ of $A$.

Proof. If $i\left(h, a^{\prime}\right)=0$ for every $a^{\prime} \in A^{\prime}$, every $a^{\prime}$ is contained in one of the two closed half planes defined by $h$, and since $A^{\prime}$ is $i$-connected, the same holds for $\operatorname{Hull}\left(A^{\prime}\right)$, implying $i(h, g)=0$ for every $g \in \Delta\left(\operatorname{Hull}\left(A^{\prime}\right)\right)$.

Let $A^{\prime}, A^{\prime \prime}$ be distinct $i-c C, g^{\prime} \in \Delta\left(\operatorname{Hull}\left(A^{\prime}\right)\right), g^{\prime \prime} \in \Delta\left(\operatorname{Hull}\left(A^{\prime \prime}\right)\right)$ and assume $i\left(g^{\prime}, g^{\prime \prime}\right)=1$. By the claim there is $a^{\prime \prime} \in A^{\prime \prime}$ with $i\left(g^{\prime}, a^{\prime \prime}\right)=1$ and hence $a^{\prime} \in A^{\prime}$ with $i\left(a^{\prime}, a^{\prime \prime}\right)=1$, a contradiction. Thus $i\left(g^{\prime}, g^{\prime \prime}\right)=0$.

Proposition 3.2. Let $A \subset \mathcal{G}(\mathbb{H})$ be a set of geodesics and $\wedge_{A}$ as defined in (3.1). Then

(1) $\Lambda_{A}$ is a lamination and $\Lambda_{A}=A^{0} \cap A^{00}$. In particular $i\left(\Lambda_{A}, A\right)=0$;

(2) for any complementary region $\mathcal{R}$ of $\Lambda_{A}$ one of the following holds

(a) either no geodesic of $A$ meets $\mathcal{R}$, or

(b) $A_{\mathcal{R}}:=\{a \in A:$ a is contained in $\mathcal{R}\}$ is an $i$-connected component of $A$, and $\mathcal{R}=\operatorname{Hull}\left(A_{\mathcal{R}}\right)$. In particular every geodesic g meeting $\mathcal{R}$ must intersect transversally some geodesic in $A_{\mathcal{R}}$.

Proof. It is immediate from Lemma 3.1 that $\Lambda_{A}$ is a lamination and $i\left(\Lambda_{A}, A\right)=$ 0 . We will prove that $\Lambda_{A}=A^{0} \cap A^{00}$ after having proven (2).

If $\mathcal{R}$ is a complementary region and $a \in A$ intersects $\mathcal{R}$ then $a \subset \mathcal{R}$ since $i\left(\Lambda_{A}, A\right)=0$. Thus, the $i$-cc-component $A^{\prime}$ of $a$ is formed of geodesics all 
in $\mathcal{R}$ and hence $\operatorname{Hull}\left(A^{\prime}\right) \subset \mathcal{R}$. Since $\Delta\left(\operatorname{Hull}\left(A^{\prime}\right)\right) \subset \Lambda_{A}$, we conclude that $\operatorname{Hull}\left(A^{\prime}\right)=\mathcal{R}$, thus proving (2).

We now complete the proof of (1). Since $i\left(\Lambda_{A}, A\right)=0$, it follows that $\Lambda_{A} \subset A^{0}$. If $i\left(\Lambda_{A}, A^{0}\right)>0$, then there is an $i$-cc $A^{\prime}$ with $i\left(\Delta\left(\operatorname{Hull}\left(A^{\prime}\right)\right), A^{0}\right)>$ 0 which by Lemma 3.1 would imply that $i\left(A^{\prime}, A^{0}\right)>0$, a contradiction. Thus $\Lambda_{A} \subset A^{0} \cap A^{00}$.

Conversely, if $g \in\left(A^{0} \cap A^{00}\right) \backslash \Lambda_{A}$, then since $A^{0} \cap A^{00}$ is a lamination, there is a complementary region $\mathcal{R}$ of $\Lambda_{\mathcal{A}}$ with $g \subset \mathcal{R}$. Since $g \in A^{0}$, if follows from (2b) and Lemma 3.1 that no geodesic of $A$ intersects $\mathcal{R}$ nontrivially and hence $\mathcal{G}(\mathcal{R}) \subset A^{0}$. Since $\mathcal{G}(\mathcal{R}) \neq \varnothing$, the region $\mathcal{R}$ must have a least four ideal vertices and hence there is $h \in \mathcal{G}(\mathcal{R}) \subset A^{0}$ with $i(g, h)=1$ implying $g \notin A^{00}$, a contradiction.

3.2. The structure of subsets of geodesics in $\Sigma$. Let now $S=\Gamma \backslash \mathbb{H}$ be a geometrically finite hyperbolic surface and $\Sigma=\Gamma \backslash C$ its convex core. The covering projection $\pi: \mathbb{H} \rightarrow S$ induces a map $\mathcal{G}(\mathbb{H}) \rightarrow \mathcal{G}(S)$ still denoted by $\pi$. For $g, h \in \mathcal{G}(S)$ we define $i(g, h)$ as the sum of $i\left(g^{\prime}, h^{\prime}\right)$ where $\left(g^{\prime}, h^{\prime}\right)$ runs through a fundamental domain for the $\Gamma$-action on $\pi^{-1}(\mathrm{~g}) \times \pi^{-1}(\mathrm{~h})$; if $\mathrm{g}$, $\mathrm{h}$ are distinct closed geodesics this recovers the usual intersection number. For subsets $A, B \subset \mathcal{G}(S)$ we extend the definition of $i$ to $i(A, B)$ as in $\$ 3.1$ and define $A^{0}$ analogously. Given $A \subset \mathcal{G}(\Sigma)$ we consider the set $\mathcal{E}_{A}$ of solitary elements among the set of closed geodesics in $A^{0}$, that is

$$
\begin{aligned}
\mathcal{E}_{A}:=\left\{c \in \mathcal{G}(\Sigma): c \text { closed, } i(A, c)=0 \text { and } i\left(c, c^{\prime}\right)=0\right. \\
\left.\forall c^{\prime} \in \mathcal{G}(\Sigma) \text { with } c^{\prime} \text { closed and } i\left(A, c^{\prime}\right)=0\right\} .
\end{aligned}
$$

Observe that if $A$ is the support of a geodesic current $\mu$ on $\Sigma, \varepsilon_{A}$ is nothing but the set $\mathcal{E}_{\mu}$ defined in the introduction. In general $\mathcal{E}_{A}$ consists of simple, closed, pairwise disjoint geodesics and contains all the boundary components of $\Sigma$. In particular $\mathcal{E}_{A}$ is a geodesic lamination and furthermore it induces a partition of $A$ :

$$
A=\left(A \cap \varepsilon_{A}\right) \sqcup \bigsqcup_{\mathcal{R}} A_{\mathcal{R}}
$$

where the disjoint union is over all complementary regions $\mathcal{R}$ of $\mathcal{E}_{\mathrm{A}}$ and $A_{\mathcal{R}}:=A \cap \mathcal{G}(\mathcal{R})$.

Proposition 3.3. Let $A$ be a subset of $\mathcal{G}(\Sigma)$, and let $\varepsilon_{A}$ be as in (3.2). Then for every complementary region $\mathcal{R}$ of $\mathcal{E}_{\mathrm{A}}$ precisely one of the following holds:

(1) either no geodesic of $A$ meets $\mathcal{R}$,

(2) or any closed geodesic $\mathrm{c} \subset \sum$ meeting $\mathcal{R}$ must intersect transversely some geodesic of $\mathrm{A}_{\mathcal{R}}$.

The proof of Proposition 3.3 uses the structure of complementary regions of a compactly supported geodesic lamination $\Lambda \subset \Sigma$ in a complete 
finite area hyperbolic surface. Namely that the complement $\Sigma \backslash \Lambda$ is a finite union of components of the following types [CEG06, Theorem I.4.2.8]:

(1) an ideal polygon;

(2) an ideal polygon containing one cusp;

(3) a totally geodesic subsurface with geodesic boundary to which one has added a crown to some boundary geodesic (such a subsurface can possibly be reduced to a single geodesic).

A crown is an infinite cylinder bounded by a geodesing on one side and by finitely many ideal sides on the other. We will need the following

Lemma 3.4. Let $\mathrm{c} \subset \Sigma \backslash \wedge$ be a geodesic bounding a crown Q. Any closed geodesic $\mathrm{c}^{\prime} \subset \Sigma$ intersecting $\mathrm{c}$ intersects a leaf of $\Lambda$.

Proof. Assume by contradiction that there exists a closed geodesic $c^{\prime}$ that intersects $c$ but doesn't intersect any leaf of $\Lambda$. We choose intersecting lifts $g, g^{\prime}$ of $c, c^{\prime}$ in $\mathbb{H}$. The lift $\widetilde{Q}$ of $\mathcal{Q}$ which is bounded by $g$ on one side is an infinite strip, bounded on the other side by countably many geodesics $l_{i} \in \widetilde{\Lambda}:=\pi^{-1}(\Lambda)$ indexed so that $l_{i}$ shares the endpoint $p_{i} \in \partial H$ with $l_{i+1}$. Since $c^{\prime}$ doesn't intersect any leaf of $\Lambda$, there exists $i$ such that $p_{i}$ is an endpoint of $g^{\prime}$. We denote by $\gamma \in \Gamma$ the hyperbolic element with axis $g^{\prime}$, and assume without loss of generality that $p_{i}=\gamma_{+}$. Then for a sufficiently high power of $\gamma, \gamma^{n}$ g intersects $l_{i}$ and $l_{i+1}$. This implies that $c$ intersects the lamination $\Lambda$, a contradiction.

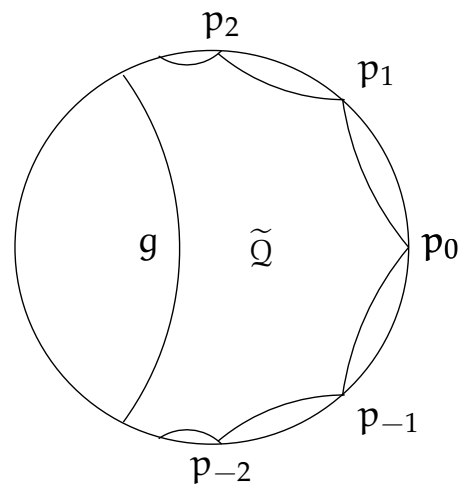

FIGURE 6. The lift $\widetilde{Q}$ of a crown is an infinite strip bound by a geodesic $\mathrm{g}$ on one side and countably many geodesics on the other side.

Proof of Proposition 3.3 Let $B=\{c \subset \Sigma$ : $c$ is a closed geodesic and $i(c, A)=$ $0\}$. We apply Proposition 3.2 to the $\Gamma$-invariant set of geodesics $\widetilde{B}:=$ $\pi^{-1}(\mathrm{~B}) \subset \mathcal{G}(\mathbb{H})$ and let $\widetilde{\Lambda}:=\Lambda_{\widetilde{B}}$ be the corresponding lamination. Set 
$\widetilde{\mathcal{E}}_{\mathrm{A}}:=\pi^{-1}\left(\mathcal{E}_{\mathrm{A}}\right)$. Since $\widetilde{\mathrm{B}} \subset \widetilde{\mathrm{B}}^{00}$, we have the following inclusion: $\widetilde{\mathcal{E}}_{\mathrm{A}}=$ $\widetilde{\mathrm{B}} \cap \widetilde{\mathrm{B}}^{0} \subset \widetilde{\mathrm{B}}^{00} \cap \widetilde{\mathrm{B}}^{0}=\widetilde{\Lambda}$.

Claim. In fact $\widetilde{\mathcal{E}}_{\mathrm{A}}=\widetilde{\Lambda}$.

Proof. $\quad$ Note that, denoting $\widetilde{A}:=\pi^{-1}(A)$, we have $\widetilde{A} \subset \widetilde{B}^{0}$, hence $\widetilde{B}^{00} \subset$ $\widetilde{A}^{0}$. Since $\widetilde{B}=\widetilde{A}^{0} \cap$ closed geodeiscs of $\Sigma$, this implies that a leaf of $\widetilde{\Lambda}$ is in $\widetilde{\mathcal{E}}_{\mathrm{A}}$ if and only if projects to a closed geodesic. Recall now that

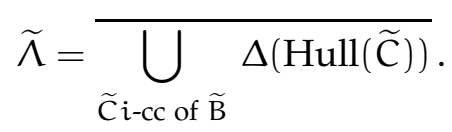

As $\widetilde{\mathcal{E}}_{\mathrm{A}}$ is closed, it is then enough to show that $\Delta(\operatorname{Hull}(\widetilde{\mathrm{C}})) \subset \widetilde{\mathcal{E}}_{\mathrm{A}}$ for all $\widetilde{\mathrm{C}}$.

Let $\widetilde{C}$ be an $i$-cc of $\widetilde{B}$ and assume first that $\widetilde{C}=\{b\}$. Then $b \in \widetilde{B}$ and $i(b, \widetilde{B})=0$, so that $b \in \widetilde{B} \cap \widetilde{B}^{0}=\widetilde{\mathcal{E}}_{A}$. Assume now that $|\widetilde{\mathrm{C}}|>1$ and let $\widetilde{Q}=\operatorname{Hull}(\widetilde{\mathrm{C}})$. Then $\mathcal{Q}:=\pi(\widetilde{\mathcal{Q}})$ is a complementary region in $\Sigma$ of the lamination $\Lambda:=\pi(\widetilde{\Lambda})$. For the sake of contradiction, assume that the boundary of $\widetilde{Q}$ contains a leaf that does not close in $\Sigma$; by the structure of complementary regions [CEG06, Theorem I.4.2.8], the projection of this leaf is then part of a crown bounded by a simple closed geodesic $\mathrm{c} \subset \Sigma$; moreover since $|\widetilde{C}|>1,2$ contains at least a pair of intersecting closed geodesics and hence the region $Q$ is not reduced to a crown, implying $c \subset Q$. Let $g \subset \widetilde{Q}$ be a lift of c. Since $i(\widetilde{A}, \widetilde{B})=0$, where $\widetilde{A}:=\pi^{-1}(A)$, Proposition 3.2 (2) implies that no geodesic in $\widetilde{A}$ meets $\widetilde{Q}$, hence $g \in \widetilde{B}$. If $c^{\prime} \subset \Sigma$ is a closed geodesic with $i\left(c^{\prime}, c\right)>0$, then for a suitable lift $g^{\prime}$ of $c^{\prime}$ we have $i\left(g^{\prime}, g\right)>0$ and thus $g^{\prime} \not \subset \widetilde{Q}$ by Lemma 3.4. This implies $g^{\prime} \notin \widetilde{B}$, hence $i\left(g^{\prime}, \widetilde{A}\right)>0$ and $i\left(c^{\prime}, A\right)>0$ which shows that $c \in \mathcal{E}_{A} \subset \Lambda$, a contradiction.

Let now $\mathcal{R}$ be a complementary region of $\Lambda$ and assume that some geodesic of $A$ intersects $\mathcal{R}$. Let $c \subset \Sigma$ be a closed geodesic intersecting $\mathcal{R}$. Let $\widetilde{\mathcal{R}}$ be a complementary region of $\widetilde{\Lambda}$ lifting $\mathcal{R}$ and $\mathrm{g}$ a lift of $\mathrm{c}$ intersecting $\widetilde{\mathcal{R}}$. Since $\widetilde{A}$ intersects $\widetilde{\mathcal{R}}$ no geodesic of $\widetilde{B}$ can intersect $\widetilde{\mathcal{R}}$ (Proposition $3.2(2)$ ) and hence $g \notin \widetilde{B}$, that is $c \notin B$, hence $i(c, A)>0$.

We now combine Proposition 3.2 and Proposition 3.3 to obtain a refined decomposition of $\Sigma$ associated with $A$ analogous to Theorem 1.2 .

Proposition 3.5. Let $A$ be a subset of $\mathcal{G}(\Sigma)$ and $\widetilde{A}=\pi^{-1}(A)$. Let $\Lambda_{\widetilde{A}}^{\text {ref }}=$ $\mathcal{E}_{A} \cup \Lambda_{A}$, where $\Lambda_{\widetilde{A}}$ is the lamination associated to $A$ by Proposition 3.2 and $\pi\left(\Lambda_{\tilde{A}}\right)=\Lambda_{\AA}$. Then $\Lambda_{\AA}^{\mathrm{ref}}$ is a lamination and we have

(1) $i\left(\Lambda_{A}^{\mathrm{ref}}, A\right)=0$; in particular

$$
A=\left(A \cap \Lambda_{\mathcal{A}}^{\mathrm{ref}}\right) \sqcup \bigsqcup_{\mathcal{R}} A_{\mathcal{R}}
$$


where $\mathcal{R}$ runs over the complementary regions of $\Lambda_{A}^{\text {ref }}$ and $A_{\mathcal{R}}:=A \cap$ $\mathcal{G}(\mathcal{R})$.

(2) For every complementary region $\mathcal{R}$ of $\Lambda_{A}^{\text {ref }}$ precisely one of the following holds:

(a) either $\mathcal{R}$ does not meet any geodesic of $A$.

(b) or every geodesic $\mathrm{g}$ of $\Sigma$ meeting $\mathcal{R}$ intersects transversely some geodesic in $\mathrm{A}$.

(3) $\wedge_{A}^{\text {ref }}$ does not meet any of the complementary regions $\mathcal{R}$ of $\mathcal{E}_{A}$ meeting no geodesic of $\mathrm{A}$.

(4) For every closed geodesic $c \subset \Sigma$, if $i(c, A)=0$, then $i\left(c, \Lambda_{A}^{\text {ref }}\right)=0$.

(5) $\mathcal{E}_{A}$ is the set of closed leaves of $\Lambda_{A}^{\text {ref. }}$

Proof. We implicitly use that the assertions of Proposition 3.2 hold true verbatim once projected to $\Sigma$.

First observe that, because of Lemma 3.1, we have $i\left(\varepsilon_{A}, \Lambda_{A}\right)=0$, hence $\Lambda_{A}^{\text {ref }}$ is a lamination. The statements (1) and (5) are clear as $\varepsilon_{A}$ and $\Lambda_{A}$ are included in $A^{0}$ by definition, and the closed geodesics in $\Lambda_{A}$ are in $\mathcal{E}_{A}$.

The dichotomy (2) is true for $\Lambda_{A}$ by Proposition 3.2, hence also for $\Lambda_{A}^{\text {ref }}$ as $\Lambda_{A}^{\text {ref }}$ refines $\Lambda_{A}$ and $\Lambda_{A}^{\text {ref }}$ is contained in $A^{0}$.

To see (3), observe that if $\mathcal{R}$ is a complementary region of $\mathcal{E}_{A}$ such that $\Lambda_{A}^{\text {ref }} \cap \mathcal{R} \neq \varnothing$, then there is a geodesic $g$ in $\Lambda_{A}$ contained in $\mathcal{R}$, since $\Lambda_{A}^{\text {ref }}$ is a lamination refining $\mathcal{E}_{\mathcal{A}}$. But then on one side of $g$ there must be a geodesic in $A$ that meets $\mathcal{R}$.

Assertion (4) follows from (3) as, by Proposition 3.3, any closed geodesic $c$ with $i(c, A)=0$ is either in $\mathcal{E}_{A}$ or contained in a complementary region $\mathcal{R}$ of $\mathcal{E}_{A}$ meeting no geodesic of $A$.

3.3. Proof of Theorem 1.2. Let $\mu$ be a geodesic current on $\Sigma$, supp $\mu \subset$ $\mathcal{G}(\mathbb{H})$ its support and $A:=\pi(\operatorname{supp} \mu)$. Observe that by definition a geodesic $g$ in $\Sigma$ is $\mu$-short if some (and hence every) lift $\widetilde{g}$ of $g$ satisfies $\mathfrak{i}(\widetilde{\mathfrak{g}}$, supp $\mu)=0$. This implies that $\mathcal{E}_{A}=\mathcal{E}_{\mu}$ and $\Lambda_{A}=A^{0} \cap A^{00}=\Lambda_{\mu}$. Then Theorem 1.2(1) follows from Proposition 3.3 and Theorem 1.2(2) follows from Proposition 3.5 .

\section{StRAight PSEUdo-DistANCE, LENGTH SHORTENING AND SYSTOLE}

The main objective of this section is to show that the systole of a current on a finite area surface $\Sigma$ with geodesic boundary can be computed using simple closed geodesics, provided $\Sigma^{\circ}$ is not the thrice punctured sphere (Corollary 4.8). This relies on two main ingredients:

(1) the fact that a geodesic current gives rise to an appropriate pseudodistance on $\mathrm{H}$ and that the length function associated to this pseudodistance behaves very much like the hyperbolic length. A similar study, in the case of closed surfaces, was carried out by Glorieux in [Glo]. 
(2) A Length-Shortening-Under-Surgery Lemma in the spirit of [MZ19], with the additional difficulty due to the presence of boundary components.

A pseudo-distance $d$ on $\mathbb{H}$ is a symmetric function $d: \mathbb{H} \times \mathbb{H} \rightarrow[0, \infty)$ vanishing on the diagonal and verifying the triangle inequality. We say that a pseudo-distance $d$ is straight if whenever three points $x, y, z$ lie on a geodesic segment on $\mathbb{H}$ (for the hyperbolic metric) in this order, we have

$$
d(x, y)+d(y, z)=d(x, z) .
$$

We emphasize that such a pseudo-distance is not necessarily continuous for the standard topology on $\mathbb{H}$.

We now turn to the construction of a straight pseudo-distance associated to a geodesic current on $\mathbb{H}$. Given a geodesic current $\mu$ on $\mathbb{H}$, define for $x, y \in \mathbb{H}$ :

$$
d_{\mu}(x, y)=\frac{1}{2}\left\{\mu\left(\mathcal{G}_{[x, y)}^{\pitchfork}\right)+\mu\left(\mathcal{G}_{(x, y]}^{\pitchfork}\right)\right\}
$$

where for a possibly empty geodesic segment $\mathrm{I} \subset \mathbb{H}$ we define

$$
\mathcal{G}_{\mathrm{I}}^{\pitchfork}=\{\mathrm{g} \in \mathcal{G}(\mathbb{H}):|g \cap \mathrm{I}|=1\} .
$$

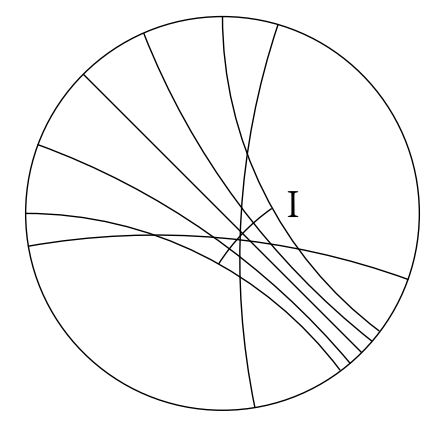

FIgURE 7. Some of the geodesics in $\mathcal{G}_{I}^{\text {}}$

Proposition 4.1. The function $\mathrm{d}_{\mu}$ is a straight pseudo-distance.

Proof. By definition $d_{\mu}$ is symmetric and vanishes on the diagonal.

In order to check the triangle inequality we may assume that the points $x, y, z$ are pairwise distinct. 
If $g \in \mathcal{G}_{[x, y)}^{\mathrm{D}}$, then either $\mathrm{g} \in \mathcal{G}_{(x, y)}^{\mathrm{x}}$ or $\mathrm{g} \cap[\mathrm{x}, \mathrm{y})=$ $\{x\}$. If $g \in \mathcal{G}_{(x, y)}^{\infty}$, then either $g \in \mathcal{G}_{[x, z)}^{\dagger}$ or $g \in \mathcal{G}_{[z, y)}^{\dagger}$. If on the other hand $g \cap[x, z)=\{x\}$, then either $g \in \mathcal{G}_{[x, y)}^{\infty}$ or otherwise $g$ contains the segment $[x, y)$ which implies that $x, y, z$ don't lie on a geodesic and hence $g \in \mathcal{G}_{[y, z)}^{\dagger}$.

This shows the inclusion

$$
\mathcal{G}_{[x, z)}^{\pitchfork} \subset \mathcal{G}_{[x, y)}^{\pitchfork} \cup \mathcal{G}_{[y, z)}^{\dagger} .
$$

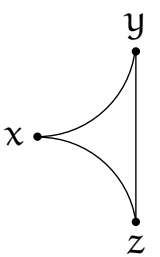

An analogous argument shows the corresponding statement for $(x, z]$ and concludes the proof of the triangle inequality.

If the three points $x, y, z$ lie on a geodesic in this order, then $\mathcal{G}_{[x, z)}^{\dagger}$ is the disjoint union of $\mathcal{G}_{[x, y)}^{\mathrm{d}}$ and $\mathcal{G}_{[y, z)}^{\mathrm{T}}$ and analogously for $(x, z]$. This implies that $d_{\mu}(x, z)=d_{\mu}(x, y)+d_{\mu}(y, z)$ and hence $d_{\mu}$ is straight.

\section{Example 4.2.}

(1) If $\mu=\mathcal{L}$ is the Liouville current on $\mathbb{H}$, the corresponding pseudodistance $d_{\mu}$ is the hyperbolic metric $d_{\text {hyp }}$.

(2) If $\mu \in \mathcal{C}(\mathbb{H})$ is a geodesic current on $\mathbb{H}$ such that $\operatorname{supp}(\mu) \subset \mathcal{G}(\mathbb{H})$ is a geodesic lamination, a standard argument shows that the quotient metric space $X_{\mu}=\mathbb{H} / \sim$, obtained by identifying points at $d_{\mu^{-}}$ distance zero, is 0-hyperbolic in the sense of Gromov and can therefore be canonically embedded in a complete $\mathbb{R}$-tree (see [MS91] for instance).

Given a straight pseudo-distance $\mathrm{d}$ on $\mathbb{H}$ we define as usual the length $\mathrm{L}(\mathrm{c})$ of a continuous path $\mathrm{c}:[\mathrm{r}, \mathrm{s}] \rightarrow \mathbb{H},-\infty<\mathrm{r} \leqslant \mathrm{s}<+\infty$ by

$$
L(c)=\sup \left\{\sum_{i=0}^{n} d\left(c\left(t_{i}\right), c\left(t_{i+1}\right)\right): n \geqslant 1, t_{0}=r \leqslant t_{1} \leqslant \cdots \leqslant t_{n}=s\right\} .
$$

Of course $\mathrm{L}(\mathrm{c})$ is invariant by monotone continuous reparametrization. The statements in the following lemma are straightforward verifications.

Lemma 4.3. The length function $\mathrm{L}$ associated to a straight pseudo-distance $\mathrm{d}$ has the following properties:

(1) If $\mathrm{c}:[\mathrm{r}, \mathrm{s}] \rightarrow \mathbb{H}$ is a continuous path, then $\mathrm{L}(\mathrm{c}) \geqslant \mathrm{d}(\mathrm{c}(\mathrm{r}), \mathrm{c}(\mathrm{s}))$ and if $\mathrm{c}$ parametrizes a geodesic segment, equality holds.

(2) If a path $\mathrm{c}$ is the concatenation $\mathrm{c}=\mathrm{c}_{1} * \mathrm{c}_{2}$ of two paths $\mathrm{c}_{1}, \mathrm{c}_{2}$, then

$$
\mathrm{L}(\mathrm{c})=\mathrm{L}\left(\mathrm{c}_{1}\right)+\mathrm{L}\left(\mathrm{c}_{2}\right) \text {. }
$$

Let now $\Gamma<\operatorname{PSL}(2, \mathbb{R})$ be a torsion-free discrete subgroup and $S:=$ $\Gamma \backslash \mathbb{H}$ be the corresponding quotient surface. Given a $\Gamma$-invariant straight 
pseudo-distance $d$ on $\mathbb{H}$ we define the length $\mathrm{L}(\mathrm{c})$ of a continuous path $c:[r, s] \rightarrow S$ as the length $\mathrm{L}(\widetilde{\mathrm{c}})$ of any continuous lift $\widetilde{\mathrm{c}}:[\mathrm{r}, \mathrm{s}] \rightarrow \mathbb{H}$.

The following generalizes a fundamental property of hyperbolic length to length functions associated to straight pseudo-distances.

Proposition 4.4. Let $\mathrm{s} \subset \mathrm{S}$ be a closed geodesic represented by a hyperbolic element $\gamma \in \Gamma$ and let $\mathrm{p}$ be a point on the axis of $\gamma$. Then for every closed loop $\mathrm{c}$ in the free homotopy class of $\mathrm{s}$

$$
\mathrm{L}(\mathrm{c}) \geqslant \mathrm{L}(\mathrm{s})=\mathrm{d}(\mathrm{p}, \gamma \mathrm{p}) .
$$

Using the definitions of the length $L$ of a curve in $S$ and Lemma4.3, the above proposition is a direct consequence of the following:

Lemma 4.5. With the hypotheses of Proposition 4.4

$$
d(q, \gamma q) \geqslant d(p, \gamma p) \text { for all } q \in \mathbb{H} .
$$

Proof. Using that $\mathrm{d}$ is straight, $\Gamma$-invariant, and applying the triangle inequality we obtain for all $n \geqslant 1$ :

$$
\begin{aligned}
n d(p, \gamma p) & =\sum_{i=0}^{n-1} d\left(\gamma^{i} p, \gamma^{i+1} p\right) \\
& =d\left(p, \gamma^{n} p\right) \\
& \leqslant d(p, q)+\sum_{i=0}^{n-1} d\left(\gamma^{i} q, \gamma^{i+1} q\right)+d\left(\gamma^{n} q, \gamma^{n} p\right) \\
& =2 d(p, q)+n d(q, \gamma q) .
\end{aligned}
$$

Dividing by $n$ and letting $n$ tend to infinity we obtain the lemma.

Assume now that $\Gamma$ is finitely generated. Let $\mathrm{C} \subset \mathbb{H}$ be the closed convex hull of the limit set of $\Gamma$ and $\Sigma=\Gamma \backslash \mathrm{C}$ be the quotient surface, which is a complete hyperbolic surface with geodesic boundary and finite area, included in $S=\Gamma \backslash \mathbb{H}$. Let $\Sigma^{\circ}$ be the interior of $\Sigma$. Define

$$
\begin{aligned}
\text { Syst }(\mathrm{L}) & :=\inf \left\{\mathrm{L}(\mathrm{c}): \mathrm{c} \subset \Sigma^{\circ} \text { is a closed geodesic }\right\} \\
\text { Syst }_{\mathrm{s}}(\mathrm{L}): & =\inf \left\{\mathrm{L}(\mathrm{c}): \mathrm{c} \subset \Sigma^{\circ} \text { is a simple closed geodesic }\right\} .
\end{aligned}
$$

Our objective is to show:

Proposition 4.6. Let $\Sigma=\Gamma \backslash C$ be a finite area surface with geodesic boundary and $\mathrm{L}$ be the length function associated to a $\Gamma$-invariant straight pseudo-distance on $\mathbb{H}$.

(1) If $\sum^{\circ}$ is not the thrice punctured sphere, $\operatorname{Syst}(\mathrm{L})=\operatorname{Syst}_{\mathrm{s}}(\mathrm{L})$.

(2) If $\sum^{\circ}$ is the thrice punctured sphere

$$
\operatorname{Syst}(\mathrm{L})=\min \left\{\mathrm{L}(\mathrm{c}): \mathrm{c} \subset \sum^{\circ} \text { is a closed geodesic with } i(\mathrm{c}, \mathrm{c})=1\right\} \text {. }
$$


Before indicating the proof of Proposition 4.6 we establish the link with the systole of a current. Let thus $\mu$ be a geodesic current on $\Sigma$, and let $d_{\mu}$ be the invariant straight pseudo-distance on $\mathbb{H}$ and $\mathrm{L}_{\mu}$ be the corresponding length function on curves in $\mathrm{S}=\Gamma \backslash \mathbb{H}$.

Lemma 4.7. Let $\mathrm{c} \subset \mathrm{S}$ be a closed geodesic. Then $\mathrm{i}(\mu, \mathrm{c})=\mathrm{L}_{\mu}(\mathrm{c})$.

Proof. Let $\gamma \in \Gamma$ be a hyperbolic element representing $\mathrm{c}$ and let $\mathrm{p}$ be a point on its axis g. Then we have (by Proposition 4.4):

$$
\mathrm{L}_{\mu}(\mathrm{c})=\mathrm{d}_{\mu}(\mathrm{p}, \gamma \mathrm{p})=\frac{1}{2}\left\{\mu\left(\mathcal{G}_{[\mathrm{p}, \gamma p)}^{\pitchfork}\right)+\mu\left(\mathcal{G}_{(\mathrm{p}, \gamma p]}^{\pitchfork}\right)\right\} .
$$

Observe that $\mathcal{G}_{[p, \gamma p)}^{\pitchfork} \times\{g\}$ as well as $\mathcal{G}_{(p, \gamma p]}^{\pitchfork} \times\{g\}$ are Borel fundamental domains for the $\Gamma$-action on $\left(\mathcal{G}(\mathbb{H}) \times \operatorname{supp} \delta_{c}\right) \cap \mathcal{D G}(\mathbb{H})$ and hence their $\mu$-measure equals $i(\mu, c)$ by definition.

Lemma 4.7 and Proposition 4.6 now lead to the main result of this section concerning the systole of $\mu$.

Recall that

$$
\operatorname{Syst}(\mu)=\inf \left\{i(\mu, c): c \text { is a closed geodesic in } \Sigma^{\circ}\right\}
$$

and

$$
\text { Syst }_{s}(\mu)=\inf \left\{i(\mu, c): c \text { is a simple closed geodesic in } \Sigma^{\circ}\right\} \text {. }
$$

Then

Corollary 4.8. Let $\mu$ be a geodesic current on a finite area hyperbolic surface $\Sigma$ with geodesic boundary.

(1) If $\sum^{\circ}$ is not the thrice punctured sphere,

$$
\operatorname{Syst}(\mu)=\operatorname{Syst}_{s}(\mu) \text {. }
$$

(2) If $\sum^{\circ}$ is the thrice punctured sphere and the carrier of $\mu$ is not included in $\partial \Sigma$

$$
\operatorname{Syst}(\mu)>0 \text {. }
$$

Proof. (1) This statement follows from Proposition 4.6(1) and Lemma 4.7.

(2) Since the carrier of $\mu$ is not contained in the boundary of $\Sigma$, there is a geodesic $g \subset \Sigma^{\circ}$ in the carrier of $\mu$. But $g$ intersects then transversally at least one closed geodesic with one self-intersection. The claim follows then from Proposition 4.6(2).

We now indicate the main steps in the proof of Proposition 4.6. It relies on a "Length-Shortening-Under-Surgery" property and standard arguments from surface topology. In the sequel we will use the notation $i\left(c, c^{\prime}\right)$ for distinct loops $c, c^{\prime}$ as the minimum intersection number of loops in the free homotopy classes represented by $c$ and $c^{\prime}$. Instead we will denote by $i(c, c)$ the number of self-intersections of $c$. 
Let now $c \subset S$ be a closed geodesic with at least one self-intersection point $p \in c$. Choose a parametrisation $c:[0,1] \rightarrow S$ and $t \in(0,1)$ such that $\mathrm{p}=\mathrm{c}(0)=\mathrm{c}(1)=\mathrm{c}(\mathrm{t})$. Then $\mathrm{c}$ is the concatenation of $\mathrm{c}_{1}=\left.\mathrm{c}\right|_{[0, \mathrm{t}]}$ and $\mathrm{c}_{2}=\left.\mathrm{c}\right|_{[\mathrm{t}, 1]}$; let also $\overline{\mathrm{c}}_{2}$ denote the loop $\mathrm{c}_{2}$ with opposite orientation and let $\mathrm{c}_{3}=\mathrm{c}_{1} * \overline{\mathrm{c}}_{2}$ be the concatenation of $\mathrm{c}_{1}$ and $\overline{\mathrm{c}}_{2}$.

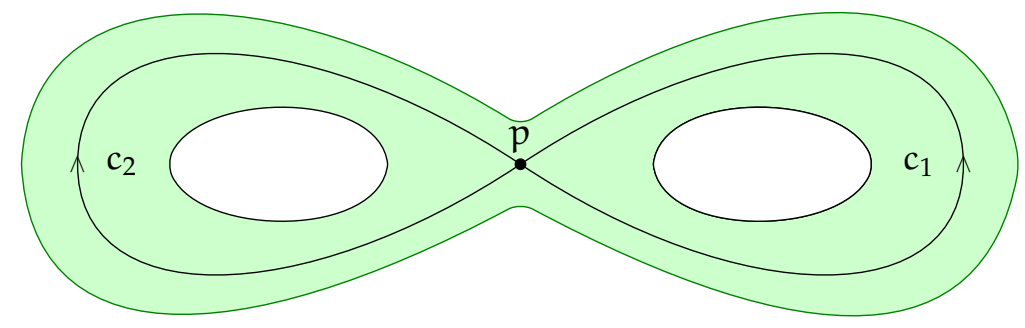

Observe that

$$
\mathrm{L}\left(\mathrm{c}_{3}\right)=\mathrm{L}\left(\mathrm{c}_{1}\right)+\mathrm{L}\left(\mathrm{c}_{2}\right)=\mathrm{L}(\mathrm{c}) \text { and } i\left(\mathrm{c}_{i}, \mathrm{c}_{i}\right)<i(\mathrm{c}, \mathrm{c}), i \in\{1,2,3\} .
$$

The following is an immediate consequence of Proposition 4.4.

Lemma 4.9. If for $i \in\{1,2,3\}$, if $\mathrm{c}_{\boldsymbol{i}}$ is freely homotopic to the closed geodesic $s_{i}$, then $\mathrm{L}\left(\mathrm{s}_{i}\right) \leqslant \mathrm{L}(\mathrm{c})$.

Given a closed geodesic $c \subset \Sigma$ with positive self-intersection, let $\Sigma_{c} \subset \Sigma$ denote the subsurface filled by $c$, that, we recall, is obtained by taking a regular tubular neighborhood of $c$ and adding to it all the components of the complement that are either simply connected or whose fundamental group is cyclic. Standard arguments in surface topology, combined with Lemma 4.9 then imply:

\section{Lemma 4.10.}

(1) If $\mathrm{c} \subset \sum$ is a closed self-intersecting geodesic, we have for every connected component $\mathrm{c}^{\prime}$ of $\partial \Sigma_{\mathrm{c}}$ :

$$
\mathrm{L}\left(\mathrm{c}^{\prime}\right) \leqslant \mathrm{L}(\mathrm{c}) .
$$

(2) Given a closed geodesic $c \subset \Sigma$ with positive self-intersection, there exists a closed geodesic $\mathrm{c}^{\prime}$ in $\Sigma_{\mathrm{c}}$ with $\mathrm{i}\left(\mathrm{c}^{\prime}, \mathrm{c}^{\prime}\right)=1$ and $\mathrm{L}\left(\mathrm{c}^{\prime}\right) \leqslant \mathrm{L}(\mathrm{c})$. In particular $\Sigma_{c^{\prime}}^{\circ}$ is a thrice punctured sphere.

Proof of Proposition 4.6

(1) Let $c \subset \sum^{\circ}$ be a closed geodesic with positive self-intersection; by Lemma 4.10 (2) there is a closed geodesic $c^{\prime}$ in $\Sigma^{\circ}$ with $i\left(c^{\prime}, c^{\prime}\right)=1$ and $\mathrm{L}\left(\mathrm{c}^{\prime}\right) \leqslant \mathrm{L}(\mathrm{c})$. Since $\stackrel{\Sigma}{\Sigma}_{\mathrm{c}^{\prime}}$ is a thrice punctured sphere and $\Sigma^{\circ}$ is not, there is a boundary component $c^{\prime \prime}$ of $\Sigma_{c^{\prime}}$ which is a simple closed geodesic contained in $\Sigma^{\circ}$; Lemma 4.10(1) implies then that $\mathrm{L}\left(\mathrm{c}^{\prime \prime}\right) \leqslant \mathrm{L}\left(\mathrm{c}^{\prime}\right) \leqslant \mathrm{L}(\mathrm{c})$ and this shows that Syst $(\mathrm{L})=$ Syst $_{\mathrm{s}}(\mathrm{L})$.

(2) Follows immediately from Lemma 4.10(2). 
Remark 4.11. Lemma 4.9 is Proposition 4.5 in [MZ19] for the case where $\mathrm{S}=\Gamma \backslash \mathbb{H}$ is compact. We believe that the use of pseudo-distance associated to a current simplifies the arguments.

\section{Currents with positive systole}

This section is devoted to the proofs of Theorem 1.3 and Corollary 1.5 .

Let then $\Gamma<\operatorname{PSL}(2, \mathbb{R})$ be finitely generated and torsion-free, $S=\Gamma \backslash \mathbb{H}$ the quotient surface and $\Sigma=\Gamma \backslash \mathrm{C}$ the corresponding finite area surface with geodesic boundary.

Let $\mu \in \mathcal{C}(\Sigma)$ be a geodesic current on $\Sigma$ and recall that a geodesic $c \subset \Sigma$ is $\mu$-short if some (and hence any) lift $\widetilde{c}$ of $c$ does not intersect transversally any geodesic in the support of $\mu$. The main ingredients in the proof of Theorem 1.3 are the results on systoles established in $\S 4$ together with the following proposition, establishing the implication (1) $\Longrightarrow(4)$ of Theorem 1.3 .

Proposition 5.1. Let $\mu$ be a geodesic current on $\Sigma$ and $c: \mathbb{R} \rightarrow \Sigma a \mu$-short geodesic that is recurrent in $\Sigma^{\circ}$. Then for all $\varepsilon>0$, there exists a closed geodesic $\mathrm{c}_{\mathcal{\varepsilon}}$ in $\sum^{\circ}$ such that $i\left(\mu, c_{\varepsilon}\right)<\varepsilon$.

The hyperbolic metric on $\mathbb{H}$ and $S$ induces Riemannian metrics on the respective unit tangent bundles $T^{1} \mathbb{H}$ and $T^{1} S$, denoted $d^{*}$, for which the projection maps are Riemannian submersions; as usual $d_{\text {hyp }}$ will denote the hyperbolic distance on $S$ and $\mathbb{H}$. We denote by $g_{t}$ the geodesic flow action on $\mathrm{T}^{1} \mathbb{H}$.

We will use:

Closing Lemma. [Ebe96, 4.5.15] Given a compact set $\mathrm{C} \subset \mathrm{T}^{1}(\mathbb{H})$ and $\zeta>0$, there exist $\mathrm{T} \geqslant 0$ and $\delta>0$ such that if there is $\mathrm{t} \geqslant \mathrm{T}, v \in \mathrm{C}$ and $\gamma \in \operatorname{PSL}(2, \mathbb{R})$ with $\mathrm{d}^{*}\left(\gamma(v), \mathrm{g}_{\mathrm{t}}(v)\right)<\delta$, then there is $\mathrm{t}^{\prime} \in \mathbb{R}$ with $\left|\mathrm{t}^{\prime}-\mathrm{t}\right|<\zeta$ and $v^{\prime} \in \mathrm{T}^{1} \mathbb{H}$ with $\mathrm{d}^{*}\left(v^{\prime}, v\right)<\zeta$ and $\gamma\left(v^{\prime}\right)=\mathrm{g}_{\mathrm{t}^{\prime}}\left(v^{\prime}\right)$.

Proof of Proposition 5.1. We may suppose that $c$ is not closed (otherwise the statement is clear). Recall that $c$ is recurrent in $\Sigma^{\circ}$ if there exists a sequence $\left(t_{n}\right)_{n \geqslant 1}$ in $\mathbb{R}$ with $\lim _{n}\left|t_{n}\right|=\infty$ and $\left\{c\left(t_{n}\right): n \geqslant 1\right\}$ stays in a fixed compact subset $\mathrm{K}$ of $\sum^{\circ}$. Modulo reparametrizing with opposite orientation, we may assume that the sequence $\left(t_{n}\right)_{n \geqslant 1}$ is monotone increasing with $\lim t_{\mathrm{n}}=+\infty$. Let $v \in \mathrm{T}_{\mathrm{p}}^{1} \sum^{\circ}$ be an accumulation point of the sequence $\left(\dot{c}\left(t_{\mathfrak{n}}\right)\right)_{n \geqslant 1}$; enlarging $\mathrm{K}$ we may assume $\mathrm{p} \in \stackrel{\circ}{\mathrm{K}}$. Let $s: \mathbb{R} \rightarrow \mathrm{S}$ be the unit speed geodesic with $\dot{\mathrm{s}}(0)=v$, and $\mathrm{g} \subset \mathbb{H}$ be a lift of $s$.

Claim. For all $\epsilon>0$, and for all $\widetilde{\mathrm{p}} \in \mathrm{g}$ except at most countably many, there exists $\eta>0$ so that $\mu\left(\mathcal{G}_{\overline{\mathrm{B}}(\widetilde{\mathrm{p}}, \eta)} \backslash\{\mathrm{g}\}\right)<\varepsilon$. 
Proof. Let $t_{0}>0$ be such that $s\left(\left(-t_{0}, t_{0}\right)\right) \subset \stackrel{\circ}{K}$. Then for any $t \in$ $\left(-t_{0}, t_{0}\right), \dot{s}(t)$ is an accumulation point of the sequence $\left(\dot{c}\left(t_{n}+t\right)\right)_{n \geqslant 1}$. Let $g \subset \mathbb{H}$ be a lift of $s$; we claim that the set of points $x \in g$ such that $\mu\left(\mathcal{G}_{\{x\}}\right)>\mu(\{g\})$ is at most countable. Indeed, the family of Borel subsets $\left\{\mathcal{G}_{\{x\}} \backslash\{g\}_{x \in g}\right.$ are pairwise disjoint; since $\mu$ is $\sigma$-finite, the claim follows. Thus replacing $p=s(0)$ by $s(t)$ for some appropriate $t \in\left(-t_{0}, t_{0}\right)$ we may

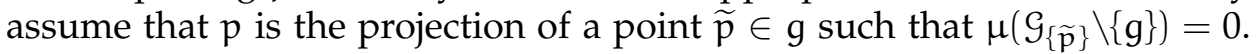
Since $\mu\left(\mathcal{G}_{\{\tilde{p}\}} \backslash\{g\}\right)=0$, we may now choose $\eta>0$ such that $\mu\left(\mathcal{G}_{\overline{\mathrm{B}}(\tilde{\mathrm{p}}, \eta)} \backslash\{g\}\right)<$ $\varepsilon$, where $\bar{B}(\widetilde{p}, \eta) \subset \mathbb{H}$ is the closed ball centered at $\widetilde{p}$ of radius $\eta$ for the hyperbolic metric.

Possibly decreasing $\eta$ we may in addition assume that $\eta$ is smaller than $d_{\text {hyp }}(p, \partial \Sigma)$ and the injectivity radius at $p$. In particular, the projection $\pi: \mathbb{H} \rightarrow S$ sends $\bar{B}(\widetilde{p}, \eta)$ isometrically to $\bar{B}(p, \eta)$, the corresponding metric ball in $S$, and $\bar{B}(p, \eta) \subset \Sigma^{\circ}$. As $c$ is not closed, $\dot{c}\left(t_{n}\right)$ is never tangent to $s$, so we may assume that $c\left(t_{n}\right)$ never belongs to $s$. Passing to a subsequence we may now assume that all points $c\left(t_{n}\right)$ are on the same side of $s$ in $\bar{B}(p, \eta)$.

Claim. If $\alpha:\left[0, \mathrm{t}_{\mathrm{n}}-\mathrm{t}_{\mathrm{m}}+1\right] \rightarrow \Sigma^{\circ}$ is a closed loop obtained by concatenation of $\left.c\right|_{\left[t_{m}, t_{n}\right]}$ and the geodesic segment joining $c\left(t_{n}\right)$ and $c\left(t_{m}\right)$ in $\bar{B}(p, \eta)$, then

$$
\mathrm{L}_{\mu}(\alpha) \leqslant \varepsilon
$$

where $\mathrm{L}_{\mu}$ is the length function corresponding to $\mu$.

Proof. We have

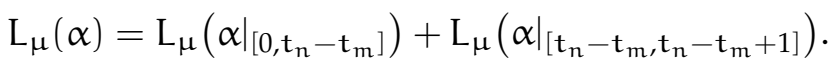

The first summand vanishes because $c$ is $\mu$-short; for the second summand observe that since $c\left(t_{n}\right)$ and $c\left(t_{m}\right)$ are on the same side of $s$ in $\bar{B}(p, \eta)$, the geodesic segment $\left[\alpha\left(t_{n}-t_{m}\right), \alpha\left(t_{n}-t_{m}+1\right)\right]$ is disjoint from $c$, contained in $\bar{B}(p, \eta)$ and as a result

$$
\mathrm{L}_{\mu}\left(\left.\alpha\right|_{\left[\mathrm{t}_{\mathfrak{n}}-\mathrm{t}_{\mathrm{m},}, \mathrm{t}_{\mathfrak{n}}-\mathrm{t}_{\mathrm{m}}+1\right]}\right) \leqslant \mu\left(\mathcal{G}_{\overline{\mathrm{B}}(\widetilde{\mathrm{p}}, \eta)} \backslash\{g\}\right)<\varepsilon .
$$

Now let $0<\zeta<\frac{\eta}{2}$ and let $C$ be the compact set consisting of unit tangent vectors based at a point of $\bar{B}\left(\widetilde{p}, \frac{\eta}{2}\right)$; let $T$ and $\delta$ be the corresponding constants given by the Closing Lemma. We may assume $\delta<\frac{\eta}{2}$ and choose $n_{0} \in \mathbb{N}$ such that $d^{*}\left(\dot{c}\left(t_{n}\right), v\right)<\frac{\delta}{2} \forall n \geqslant n_{0}$. We can pick $n>m \geqslant n_{0}$ so that $t_{n}-t_{m} \geqslant T$ and we have

$$
\mathrm{d}^{*}\left(\dot{\mathrm{c}}\left(\mathrm{t}_{\mathrm{n}}\right), \dot{\mathrm{c}}\left(\mathrm{t}_{\mathrm{m}}\right)\right)<\delta
$$

Let $\alpha:\left[0, t_{n}-t_{m}+1\right] \rightarrow \Sigma^{\circ}$ be the closed loop obtained by concatenation of $\left.c\right|_{\left[t_{m}, t_{n}\right]}$ and the geodesic segment joining $c\left(t_{n}\right)$ and $c\left(t_{m}\right)$ in $\bar{B}(p, \eta)$. Let $\widetilde{\alpha}:\left[0, t_{n}-t_{m}+1\right] \rightarrow \mathbb{H}$ be the unique lift with $d(\widetilde{\alpha}(0), \widetilde{p})<\eta$ and let $\gamma \in \Gamma$ be such that $\gamma \widetilde{\alpha}(0)=\widetilde{\alpha}\left(t_{n}-t_{m}+1\right)$. Then it follows from (5.1) that

$$
\mathrm{d}^{*}\left(\gamma \dot{\widetilde{\alpha}}(0), \dot{\widetilde{\alpha}}\left(\mathrm{t}_{\mathrm{n}}-\mathrm{t}_{\mathrm{m}}\right)\right)<\delta
$$


and hence it follows from the Closing Lemma that $\gamma$ is hyperbolic and that its axis contains a point $\widetilde{p}^{\prime}$ with $d_{\text {hyp }}\left(\widetilde{\alpha}(0), \widetilde{p}^{\prime}\right)<\zeta$, in particular $\widetilde{p}^{\prime} \in$ $\bar{B}(\widetilde{p}, \eta)$. The projection to $S$ of the axis of $\gamma$ gives us then a closed geodesic $c_{\varepsilon}$ contained in $\Sigma^{\circ}$ and for which $i\left(\mu, c_{\varepsilon}\right) \leqslant L_{\mu}(\alpha)<\epsilon$ (see Proposition 4.4 and Lemma 4.7). This concludes the proof.

Proof of Theorem 1.3 We will show that the contrapositions of properties (1), (2), (3), (4), denoted (1)', (2)', (3)', (4)', are equivalent.

$(1)^{\prime} \Longrightarrow(2)^{\prime}$ : Assume $\operatorname{Syst}(\mu)=0$. Since $\Sigma^{\circ}$ is not the thrice punctured sphere, we have $\operatorname{Syst}_{s}(\mu)=0$ by Corollary $4.8(1)$. Let thus $\left(c_{n}\right)_{n \geqslant 1}$ be a sequence of simple closed geodesics in $\sum^{\circ}$ with $\lim _{n \rightarrow \infty} i\left(\mu, c_{n}\right)=0$, in particular

$$
\lim _{n \rightarrow \infty} i\left(\mu, \frac{c_{n}}{\ell_{\text {hyp }}\left(c_{n}\right)}\right)=0
$$

Now the sequence of currents $\left(\frac{\delta_{\mathcal{c}_{n}}}{\ell_{\text {hyp }}\left(\boldsymbol{c}_{\mathfrak{n}}\right)}\right)_{n \geqslant 1}$ is contained in $\left\{v \in \mathcal{M} \mathcal{L}_{\mathcal{c}}\left(\Sigma^{\circ}\right)\right.$ : $i(\mathcal{L}, v)=1\}$. Since the latter space is compact (see Proposition 2.6 and the remark preceding Theorem 1.3), this sequence has a accumulation point, say $v_{0}$, in $\mathcal{M} \mathcal{L}_{\mathrm{c}}\left(\Sigma^{\circ}\right)$ for which $i\left(\mu, v_{0}\right)=0$ and $i\left(\mathcal{L}, v_{0}\right)=1$, in particular $v_{0} \neq 0$. This shows the announced implication.

$(2)^{\prime} \Longrightarrow(3)^{\prime}$ : clear.

$(3)^{\prime} \Longrightarrow(4)^{\prime}$ : let $v \in \mathcal{C}_{K}(\Sigma)$, with $i(\mu, v)=0$ and $v \neq 0$. Then any geodesic $g \in \operatorname{supp} v$ does not intersect transversally any geodesic of supp $\mu$; such a geodesic $\mathrm{g}$ is $\mu$-short by definition and recurrent since $\pi(\mathrm{g}) \subset \mathrm{K} \subset \Sigma^{\circ}$.

$(4)^{\prime} \Longrightarrow(1)^{\prime}:$ This is the content of Proposition 5.1

Proof of Corollary 1.5. (1) If $\Sigma^{\circ}$ is the thrice punctured sphere the assertion follows from Proposition 4.6(2). We may hence assume that $\Sigma^{\circ}$ is not the thrice punctured sphere. Let $\left(\mu_{n}\right)_{n \geqslant 1}$ be a convergent sequence in $\mathcal{C}(\Sigma)$ with limit $\mu$. Since $\lim _{n \rightarrow \infty} i\left(\mu_{n}, c\right)=i(\mu, c)$ for every closed geodesic $c$, then $\overline{\lim }_{n \rightarrow \infty} \operatorname{Syst}\left(\mu_{n}\right) \leqslant \operatorname{Syst}(\mu)$ and hence Syst is continuous if $\operatorname{Syst}(\mu)=$ 0 .

Let $\operatorname{Syst}(\mu)>0$ and assume by contradiction that

$$
\underline{\lim }_{n} \operatorname{Syst}\left(\mu_{n}\right)<\operatorname{Syst}(\mu) \text {. }
$$

For every $n \geqslant 1$, it follows from Proposition 4.6(1) that there exists a simple closed geodesic $c_{n}$ with

$$
i\left(\mu_{n}, c_{n}\right) \leqslant \operatorname{Syst}\left(\mu_{n}\right)+\frac{1}{n} .
$$

If $\left\{\ell_{\text {hyp }}\left(c_{n}\right): n \geqslant 1\right\}$ is unbounded, without loss of generality we may assume that $\lim _{n \rightarrow \infty} \ell_{\text {hyp }}\left(c_{n}\right)=\infty$ and that the sequence $\delta_{c_{n}} / \ell_{\text {hyp }}\left(c_{n}\right)$ 
converges to a compactly supported measured lamination $v \in \mathcal{M} \mathcal{L}_{\mathrm{c}}\left(\Sigma^{\circ}\right)$. But then

$$
i(\mu, v)=\lim _{n \rightarrow \infty} \frac{i\left(\mu_{n}, v_{n}\right)}{\ell_{\text {hyp }}\left(c_{n}\right)}=0,
$$

which, by Theorem 1.3, implies that $\operatorname{Syst}(\mu)=0$, a contradiction.

Hence, by passing to a subsequence, we may assume that $c_{n}=c$ for all $n \geqslant 1$, and thus

$$
\operatorname{Syst}(\mu) \leqslant i(\mu, c)=\lim _{n \rightarrow \infty} i\left(\mu_{n}, c_{n}\right) \leqslant \underline{\lim }_{n} \operatorname{Syst}\left(\mu_{n}\right),
$$

which is a contradiction.

(2) Assume that the first inequality does not hold. Then there is a sequence of closed geodesics $\left(c_{n}\right)_{n} \geqslant$ contained in $K$ such that

$$
\lim _{n \rightarrow \infty} \frac{i\left(\mu, c_{n}\right)}{\ell_{\text {hyp }}\left(c_{n}\right)}=0 \text {. }
$$

Using that

$$
\left\{\frac{\delta_{c_{n}}}{\ell_{\text {hyp }}\left(c_{n}\right)}: n \geqslant 1\right\}
$$

is relatively compact (see Proposition 2.6(1)), let $v \in \mathrm{C}_{k}(\Sigma)$, for $v \neq 0$, be an accumulation point of this sequence. Then $i(\mu, v)=0$, which contradicts Theorem 1.3(3). An analogous argument leads to the second inequality.

(3) Let $\varphi_{n}: \Sigma \rightarrow \Sigma$ be a sequence of homeomorphisms fixing pointwise $\partial \Sigma$ and such that $\varphi_{n} \rightarrow \infty$ in the mapping class group of $\Sigma$. Since $\Omega$ is locally compact, it suffices to show that if $\tau \in \Omega$ and $[\mu] \in \mathbb{P C}(\Sigma)$ is any accumulation point of $\left[\varphi_{n}(\tau)\right]$, then $\operatorname{Syst}(\mu)=0$. Let $c \subset \Sigma^{\circ}$ be a closed geodesic such that

$$
\lim _{n \rightarrow \infty} \ell_{\text {hyp }}\left(\left\{\varphi_{n}^{-1}(\mathrm{c})\right\}\right)=\infty,
$$

where $\left\{\varphi_{n}^{-1}(c)\right\}$ is the closed geodesic in the free homotopy class of $\varphi_{n}^{-1}(c)$. Then it follows from the first inequality in (2) that

$$
\lim i\left(\varphi_{n}(\tau), c\right)=\lim i\left(\tau,\left\{\varphi_{n}^{-1}(c)\right\}\right)=\infty .
$$

Let $\left(n_{k}\right)_{k \geqslant 1}$ be a subsequence and $\lambda_{k}>0$ such that

$$
\lim _{k \rightarrow \infty} \frac{\varphi_{n_{k}}(\tau)}{\lambda_{k}}=\mu
$$

In particular we have that

$$
\lim _{k \rightarrow \infty} \frac{i\left(\varphi_{n_{k}}(\tau), c\right)}{\lambda_{k}}=i(\mu, c)
$$

which implies that $\lim \lambda_{k}=\infty$. using the continuity of the systole map, we get that

$$
\operatorname{Syst}(\mu)=\lim _{k} \frac{\operatorname{Syst}\left(\varphi_{n_{k}}(\tau)\right)}{\lambda_{k}}=\lim _{k} \frac{\operatorname{Syst}(\tau)}{\lambda_{k}}=0 .
$$




\section{CuRrents With VANishing SYSTOLES AND LAMINATIONS}

In this section we establish Theorem 1.7 which characterizes geodesic currents with vanishing systole that occur as components in the decomposition theorem.

The main tools are Theorem 1.3 and the following proposition that is of independent interest.

Proposition 6.1. Let $\mu \in \mathcal{C}(\Sigma)$ and $\Lambda \subset \Sigma$ be a geodesic lamination without isolated leaves and consisting of $\mu$-short geodesics. Then for any closed geodesic $c \subset \Sigma$ bounding a crown of a complementary region of $\Lambda$ we have

$$
i(\mu, c)=0 .
$$

Let $\tilde{\Lambda}$ be the lift $\Lambda$ to a $\Gamma$-invariant geodesic lamination of $\mathbb{H}$ and let $\mathcal{R}$ be a complementary region of $\tilde{\Lambda}$. Then $\mathcal{R}$ is bounded by leaves of $\tilde{\Lambda}$ whose endpoints in $\partial \mathbb{H}$ are the vertices of $\mathcal{R}$. We now make the following crucial observation: let $a, b, c$ be consecutive vertices of $\mathcal{R}$ ordered such that $(a, b, c)$ is positively oriented; since $\Lambda$ has no isolated leaf, the pencil $\{b\} \times I_{[a, c]}$ does not contain the axis of a hyperbolic element; in addition, $b$ is in the limit set of $\Gamma$ and cannot be a cusp since otherwise $\Lambda$ would have an isolated leaf. Therefore, the hypothesis of Lemma 2.5 are fulfilled and hence

$$
\mu\left(\{b\} \times \mathrm{I}_{[\mathrm{a}, \mathrm{c}]}\right)=0 .
$$

Lemma 6.2. Let $x_{0}, \ldots, x_{n}$ be a sequence of consecutive vertices of a complementary region $\mathcal{R}$ labelled in such a way that $\left(x_{0}, \ldots, x_{n}\right)$ is positively oriented. Then the geodesic $\left(\mathrm{x}_{0}, \mathrm{x}_{\mathrm{n}}\right)$ is $\mu$-short.

Proof. The proof proceeds by recurrence. For $n=1$ the statement holds. Let us now suppose $n \geqslant 2$. We have the following equalities:

$$
\begin{aligned}
& \mathrm{I}_{\left(x_{0}, x_{n}\right)}=\mathrm{I}_{\left(x_{0}, x_{n-1}\right]} \cup \mathrm{I}_{\left(x_{n-1}, x_{\mathfrak{n}}\right)} \\
& \mathrm{I}_{\left(x_{n}, x_{0}\right)}=I_{\left(x_{n-1}, x_{0}\right)} \cap \mathrm{I}_{\left(x_{n}, x_{n-2}\right)} \cap I_{\left(x_{n}, x_{n-1}\right)} .
\end{aligned}
$$

Thus

$$
\begin{aligned}
\mathrm{I}_{\left(x_{0}, x_{n}\right)} & \times \mathrm{I}_{\left(x_{n}, x_{0}\right)} \subset \mathrm{I}_{\left(x_{0}, x_{n-1}\right)} \times \mathrm{I}_{\left(x_{n-1}, x_{0}\right)} \\
& \cup\left\{x_{n-1}\right\} \times I_{\left(x_{n}, x_{n-2}\right)} \\
& \cup I_{\left(x_{n-1}, x_{n}\right)} \times I_{\left(x_{n}, x_{n-1}\right)} .
\end{aligned}
$$

Using that $\left(x_{n-1}, x_{n}\right)$ is $\mu$-short, the induction hypothesis that $\left(x_{0}, x_{n-1}\right)$ is $\mu$-short and the observation preceding Lemma 6.2, we get to the conclusion that $\left(x_{0}, x_{n}\right)$ is $\mu$-short.

Proof of Proposition 6.1 Let $\mathcal{C} \subset \Sigma$ be a crown in the complement of the lamination $\bar{\Lambda}$, and let $\gamma \in \Gamma$ be a geodesic bounding $\mathcal{C}$. We choose lifts to 
$\mathbb{H}^{2}$ in such a way that the half plane to the left of $\left(\gamma_{+}, \gamma_{-}\right)$contains a lift $\widetilde{\mathcal{C}}$ of the crown $\mathcal{C}$.
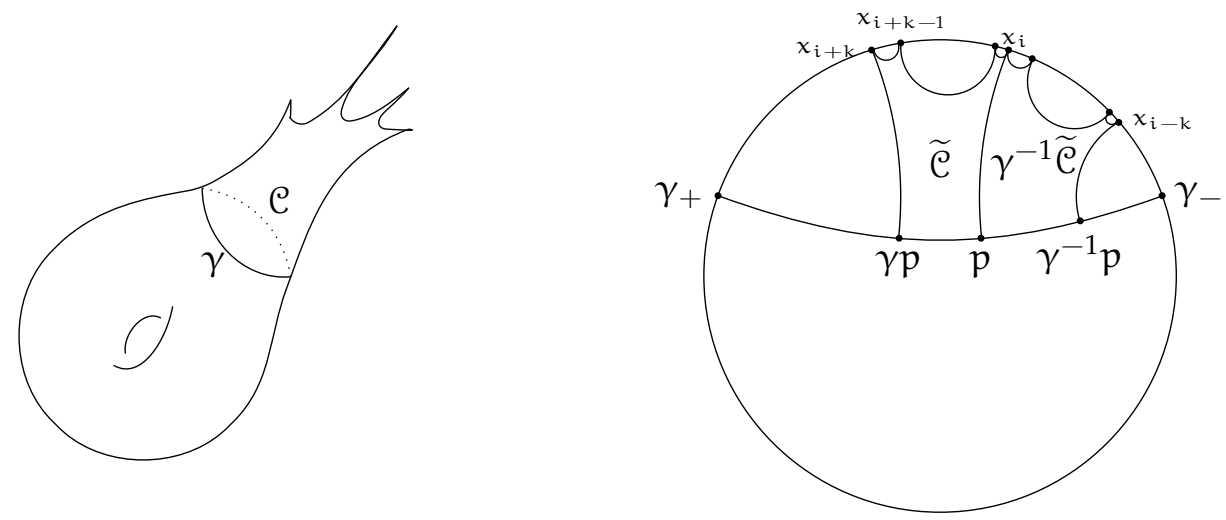

Then $\widetilde{\mathcal{C}}$ has consecutive ideal sides $\left(x_{i}, x_{i+1}\right), i \in \mathbb{Z}$, labelled in such a way that $\left(x_{i}, x_{i+1}, x_{i+2}\right)$ is positively oriented. Now observe that

$$
\left(\gamma_{-}, \gamma_{+}\right)=\lim _{n \rightarrow \infty}\left(x_{-n}, x_{n}\right)
$$

By Lemma 6.2 $\left(x_{-n}, x_{n}\right)$ is $\mu$-short, so that $\left(\gamma_{-}, \gamma_{+}\right)$is $\mu$-short since the set of $\mu$-short geodesics is a closed subset of $\mathcal{G}(\mathbb{H})$.

Proof of Theorem 1.7 Let $\mu_{\Sigma}$ be a geodesic current as in the statement of Theorem 1.7 and in the remark preceding it.

$(2) \Longrightarrow(1)$ : Follows from Theorem 1.3 since $\mu_{\Sigma} \in \mathcal{M} \mathcal{L}_{\mathcal{c}}\left(\Sigma^{\circ}\right)$ and $i\left(\mu, \mu_{\Sigma}^{\circ}\right)=0$.

$(1) \Longrightarrow(2)$ : Since $\operatorname{Syst}(\mu)=0$, Theorem 1.3 implies the existence of $v \in$ $\mathcal{M} \mathcal{L}_{\mathrm{c}}(\stackrel{\circ}{\Sigma})$ with $v \neq 0$ and $i(\mu, v)=0$. Let $\widetilde{\Lambda}=$ supp $v$ be the corresponding geodesic lamination and observe that it consists of $\mu$-short geodesics. The projection $\Lambda$ of $\tilde{\Lambda}$ to $\Sigma$ is a compact subset of $\Sigma^{\circ}$ by hypothesis. An isolated leaf $c$ of $\Lambda$ is necessarily a closed geodesic: but $c \subset \Sigma^{\circ}$ and by assumption $i(\mu, c)>0$, hence $c$ cannot be $\mu$-short. Thus $\Lambda$ has no isolated leaves. Let $\Lambda^{\prime}$ be a minimal component of $\Lambda$; then $\Lambda^{\prime}$ satisfies all the assumptions of Proposition 6.1 and since $i(\mu, c)>0$ for every closed geodesic $c \subset \Sigma^{\circ}$ we deduce that a complementary region of $\Lambda^{\prime}$ in $\Sigma$ is either an ideal polygon, an ideal polygon containing one cusp, or an ideal polygon bounding a component of $\partial \Sigma$ [CEG06. Theorem I.4.2.8]. We show now how this fact implies that $\operatorname{supp}\left(\mu_{\Sigma}\right)=\widetilde{\Lambda^{\prime}}$ where $\widetilde{\Lambda^{\prime}}$ is the lift of $\Lambda^{\prime}$ to $\mathbb{H}$.

Let $g \in \operatorname{supp}\left(\mu_{\Sigma}^{\circ}\right)$ and assume that $\mathrm{g}$ is not a leaf of $\widetilde{\Lambda}^{\prime}$. Since all leaves of $\widetilde{\Lambda}^{\prime}$ are $\mu$-short, g cannot intersect transversally a leaf of $\tilde{\Lambda}^{\prime}$, hence it is contained in a complementary region $\widetilde{\mathcal{R}}$ of $\widetilde{\Lambda}^{\prime}$. The specific structure of $\widetilde{\mathcal{R}}$ implies that if $a, b$ are the endpoints of $g$, one of $a, b$ has to be a vertex of $\widetilde{\mathcal{R}}$. If $\widetilde{\mathcal{R}}$ corresponds to a complementary region of $\Lambda^{\prime}$ bounding a cusp or a crown, it has infinitely many vertices and if it is an ideal polygon it 
must have at least four vertices since $\mathrm{g}$ is not a side of $\widetilde{\mathcal{R}}$. In any case we can find a geodesic $\delta$ connecting two vertices of $\widetilde{\mathcal{R}}$ and intersecting $\mathrm{g}$ in one point. By Lemma 6.2, $\delta$ is $\mu$-short and this contradicts the assumption that $g \in \operatorname{supp} \mu_{\Sigma}$. Thus $\operatorname{supp}\left(\mu_{\Sigma}\right) \subset \widetilde{\Lambda}^{\prime}$ and by minimality of $\Lambda^{\prime}$ we have equality.

\section{ON THE WEYL CHAMBER LENGTH COMPACTIFICATION}

Let $\Gamma<\operatorname{PSL}(2, \mathbb{R})$ be a cocompact lattice and $\rho: \Gamma \rightarrow \mathrm{G}$ a representation. Recall that when $G=\operatorname{PSL}(n, \mathbb{R}), \rho$ is Hitchin if it lies in the connected component of $\operatorname{Hom}(\Gamma, \operatorname{PSL}(n, \mathbb{R}))$ containing the restriction to $\Gamma$ of the irreducible $\operatorname{PSL}(n, \mathbb{R})$-representation of $\operatorname{PSL}(2, \mathbb{R})$, while if $G=\operatorname{Sp}(2 \mathrm{~m}, \mathbb{R})$ it is maximal if the restriction of $\rho$ to some (and hence any) torsion-free subgroup of finite index is maximal (see [BIW10, BIW14] for the relevant facts concerning maximal representations). The space $X(\Gamma, \mathrm{G})$ is then the topological space obtained by taking the quotient by $\mathrm{G}$-conjugation of the set of representations that are Hitchin if $G=\operatorname{PSL}(n, \mathbb{R})$ or maximal if $\mathrm{G}=\operatorname{Sp}(2 \mathrm{~m}, \mathbb{R})$.

Let $\lambda: G \rightarrow \mathfrak{C}$ be the Jordan projection on a closed Weyl chamber $\mathfrak{C}$ and let $\mathcal{L}: \mathcal{X}(\Gamma, G) \rightarrow \mathbb{P}\left(\mathfrak{C}^{\Gamma}\right)$ be defined by $\mathcal{L}([\rho]):=[\lambda \circ \rho]$, where $[\rho]$ refers to the G-conjugacy class of $\rho$, while $[\lambda \circ \rho]$ is the projective class of the length function $\lambda \circ \rho: \Gamma \rightarrow \mathfrak{C}$. The Weyl chamber length boundary of $\mathcal{X}(\Gamma, \mathrm{G})$ is then defined by

$$
\partial \mathcal{X}(\Gamma, \mathrm{G}):=\bigcap_{\mathrm{K}} \overline{\mathcal{L}(\mathcal{X}(\Gamma, \mathrm{G}) \backslash \mathrm{K})},
$$

where the intersection is over all compact subsets $\mathrm{K} \subset \mathcal{X}(\Gamma, \mathrm{G})$ (see [Par12]). For our purposes we make the following choices of Weyl chamber and describe the corresponding Jordan projection as well as the specific norm we use:

(1) If $G=\operatorname{PSL}(n, \mathbb{R})$, $\mathfrak{C}=\left\{\left(x_{1}, \ldots, x_{n}\right) \in \mathbb{R}^{n}: x_{1} \geqslant \cdots \geqslant x_{n}\right.$ and $\left.x_{1}+\cdots+x_{n}=0\right\}$

and

$$
\lambda(g)=\left(\ln \left|a_{1}\right|, \ldots, \ln \left|a_{n}\right|\right),
$$

where $a_{1}, \ldots, a_{n}$ are the eigenvalues of $g$ counted with multiplicity. Then for $\left(x_{1}, \ldots, x_{n}\right) \in \mathfrak{C}$,

$$
\left\|\left(x_{1}, \ldots, x_{n}\right)\right\|:=x_{1}-x_{n} .
$$

(2) If $G=\operatorname{Sp}(2 m, \mathbb{R})$,

$$
\mathfrak{C}=\left\{\left(x_{1}, \ldots, x_{m}\right) \in \mathbb{R}^{m}: x_{1} \geqslant \cdots \geqslant x_{m} \geqslant 0\right\}
$$


and $\lambda(g)$ is defined as in (7.2), where here however $a_{1}, \ldots, a_{m}$ are the eigenvalues of $g$ of absolute value $\geqslant 1$. If $\left(x_{1}, \ldots, x_{m}\right) \in \mathfrak{C}$, then

$$
\left\|\left(x_{1}, \ldots, x_{m}\right)\right\|:=\sum_{i=1}^{m} x_{i} .
$$

We will make crucial use of the results in [MZ19] that establish a relation between length functions and geodesic currents. In fact, fix $\Gamma_{0} \triangleleft \Gamma$ a torsion-free normal subgroup of finite index, and $S=\Gamma_{0} \backslash \mathbb{H}$. Then $\Gamma$ acts on the space $\mathcal{C}(S)$ of currents on $S$; the action factors via the finite group $\Gamma / \Gamma_{0}$ and the space of $\Gamma$-invariant currents $\mathcal{C}(S){ }^{\Gamma}$ is a closed subset of $\mathcal{C}(S)$. The following is a direct consequence of [MZ19]:

Corollary 7.1. For every $[\rho] \in \mathcal{X}(\Gamma, \mathrm{G})$ there is a unique current $\mu_{\rho} \in \mathcal{C}(S)^{\Gamma}$ such that for every $\gamma \in \Gamma_{0}$

$$
i\left(\mu_{\rho}, c\right)=\|\lambda(\rho(\gamma))\|,
$$

where $\mathrm{c} \subset \mathrm{S}$ is the closed geodesic corresponding to $\gamma$.

Proof. Assume $\rho: \Gamma \rightarrow \operatorname{PSL}(n, \mathbb{R})$ is Hitchin. Then $\left.\rho\right|_{\Gamma_{0}}: \Gamma_{0} \rightarrow \operatorname{PSL}(n, \mathbb{R})$ is Hitchin as well and there exists a unique $\left.\rho\right|_{\Gamma_{0}}$-invariant Frenet curve $\xi: \partial \mathbb{H} \rightarrow \mathcal{F}\left(\mathbb{R}^{n}\right)$ into the variety of full flags (see [MZ19, Definition 3.2] and [Lab06, Theorem 4.1]). But then, given any $\eta \in \Gamma$, the assignment $x \mapsto \rho(\eta)^{-1} \xi(\eta x)$ is $\left.\rho\right|_{\Gamma_{0}}$-equivariant Frenet as well, and hence coincides with $\xi$. The current $\mu$ associated to $\left.\rho\right|_{\Gamma_{0}}$ is uniquely determined by its value on rectangles, that is whenever $(x, y, z, w)$ is a positive 4-tuple in $\partial \mathbb{H}$, then

$$
\mu\left(I_{[x, y]} \times I_{[z, w]}\right)=\frac{1}{2}\{\ln [\xi(x), \xi(y), \xi(z), \xi(w)]+\ln [\xi(z), \xi(w), \xi(x), \xi(y)]\},
$$

where $[\cdot, \cdot, \cdot, \cdot]$ is a specific $\operatorname{PGL}(n, \mathbb{R})$-invariant of 4-tuples of complete pairwise transverse flags (see [MZ19, Lemma 3.6]). This, together with the $\Gamma$-equivariance of $\xi$, implies that $\mu$ is $\Gamma$-invariant.

The argument for maximal representations is completely analogous by using the continuous $\left.\rho\right|_{\Gamma_{0}}$-equivariant map $\xi: \partial \mathbb{H} \rightarrow \mathcal{L}\left(\mathbb{R}^{2 n}\right)$ into the space of Lagrangians that sends positive triples to positive triples. One concludes by uniqueness that $\xi$ is $\Gamma$-equivariant, which implies that the current $\mu$ associated to $\left.\rho\right|_{\Gamma_{0}}$ (see [MZ19, § 3.2]) is $\Gamma$-invariant.

Proof of Corollary 1.10. Observe that any $[\mathrm{L}] \in \overline{\mathcal{L}(\mathcal{X}(\Gamma, \mathrm{G}))} \subset \mathbb{P}\left(\mathfrak{C}^{\Gamma}\right)$ is homogeneous, namely $\mathrm{L}\left(\gamma^{\mathrm{k}}\right)=\mathrm{kL}(\gamma)$ for $k \in \mathbb{N}$. As a result, $\left.\mathrm{L}\right|_{\Gamma_{0}}$ does not vanish identically and the map

$$
\begin{aligned}
\mathcal{R}: \overline{\mathcal{L}(X(\Gamma, \mathrm{G}))} & \rightarrow \mathbb{P}\left(\mathbb{R}_{\geqslant 0}^{\Gamma_{0}}\right) \\
{[\mathrm{L}] } & \mapsto\left[\left.\mathrm{L}\right|_{\Gamma_{0}}\right]
\end{aligned}
$$

is well defined and continuous. 
The map I : $\mathbb{P}(\mathcal{C}(S)) \rightarrow \mathbb{P}\left(\mathbb{R}_{\geqslant 0}^{\Gamma_{0}}\right)$ that to a projectivized current associates its projectivized intersection function is a homeomorphism onto its image [Ota90], and by [MZ19, Theorem 1.1, Theorem 3.4 and Corollary 3.11], its image contains $\mathcal{R}(\mathcal{L}(\mathcal{X}(\Gamma, \mathrm{G})))$ and hence $\mathcal{R}(\overline{\mathcal{L}(\mathcal{X}(\Gamma, \mathrm{G}))})$. Thus

$$
\Omega(\Gamma, \mathrm{G})=\mathcal{R}^{-1}(\mathrm{I}(\Omega(\mathrm{S}))) \cap \partial X(\Gamma, \mathrm{G})
$$

and the assertions of Corollary 1.10 follow from the corresponding ones in Corollary 1.5 .

Next we show how Corollary 1.11 can be deduced from Theorem 1.13 and [ALS18, Theorem B]. If $\Delta=\Delta(3,3,4)$ and $G=\operatorname{PSL}(n, \mathbb{R})$ with $n \geqslant 3$ or $\mathrm{G}=\operatorname{Sp}(2 \mathrm{~m}, \mathbb{R})$ with $\mathrm{m} \geqslant 3$, then $X(\Delta, \mathrm{G})$ is a positive dimensional cell, in particular $\partial X(\Delta, G) \neq \varnothing$. Since $\Delta$ contains a torsion-free subgroup of index 24 representing a genus 2 surface, any $\Gamma$ as in Corollary 1.11 is isomorphic to a torsion-free subgroup of $\Delta$ of finite index and Theorem 1.13 implies that $\Omega(\Gamma, \mathrm{G}) \neq \varnothing$.

In the case of $\operatorname{Sp}(4, \mathbb{R})$ one can take $\Delta=\Delta(3,4,4)$; then $X(\Delta, \operatorname{Sp}(4, \mathbb{R})$ is non-compact as it contains the $\operatorname{Sp}(4, \mathbb{R})$ Hitchin component that is homeomorphic to $\mathbb{R}^{2}$ by [ALS18, Thm B], $\Delta$ contains a torsion-free subgroup of index 12 representing a genus 2 surface and the same argument as above allows us to conclude. Theorem 1.13 is in turn an immediate consequence of Corollary 7.1 and the following:

Theorem 7.2. Let $\Delta<\operatorname{PSL}(2, \mathbb{R})$ be a hyperbolic triangle group, $\Gamma<\Delta a$ torsion-free subgroup of finite index and $\mathrm{S}=\Gamma \backslash \mathbb{H}$. Then for any non-vanishing current $\mu \in \mathcal{C}(\mathrm{S})^{\Delta}$,

$$
\operatorname{Syst}(\mu)>0 \text {. }
$$

Proof. First we show that if $\tau \in \mathcal{C}(S)^{\Delta}$ with $\tau \neq 0$, then as current on $S$ it cannot be a measured lamination. Otherwise let $\mathcal{T}(\tau)$ be the $\mathbb{R}$-tree dual to $\tau$. Since $\tau$ is $\Delta$-invariant, $\Delta$ acts by isometries on the complete $\mathbb{R}$-tree $\mathcal{T}(\tau)$. The subset $\mathcal{T}(\tau)^{\mathrm{a}}$ and $\mathcal{T}(\tau)^{\mathrm{b}}$ of a-fixed, respectively b-fixed, points are not empty. Since $\mathcal{T}(\tau)$ is complete and ab has a fixed point in $\mathcal{T}(\tau)$, this implies that $\mathcal{T}(\tau)^{\mathrm{a}} \cap \mathcal{T}(\tau)^{\mathrm{b}} \neq \varnothing$ and hence $\Delta$ has a fixed point in $\mathcal{T}(\tau)$.

On the other hand pick $\gamma \in \Gamma$ representing a closed geodesic $c$ in $S$ with $i(\tau, c)>0$. Then in the tree $\mathcal{T}(\tau)$ the element $\gamma$ acts as a translation of length $i(\tau, c)$ along its axis. This is in contradiction with the fact that $\gamma$ has a fixed point.

Now let $\mu \in \mathcal{C}(S)^{\Delta}, \mu \neq 0$. Then the set $\varepsilon_{\mu}$ of closed $\mu$-short solitary geodesics is $\Delta$-invariant, and hence $\tau=\sum_{c \in \mathcal{E}_{\mu}} \delta_{\mathrm{c}}$ is a $\Delta$-invariant measured lamination on $S$, which implies that $\varepsilon_{\mu}=\varnothing$. Since $\mu \neq 0$, Theorem 1.2(1) implies that $i(\mu, c)>0$ for every closed geodesic $c \subset S$. If now $\operatorname{Syst}(\mu)=0$, Theorem 1.7 implies that $\mu$ is a measured lamination, which is impossible by the preceding discussion. Hence Syst $(\mu)>0$, which concludes the proof. 
Proof of Corollary 1.12 Let $\left(\left[\rho_{k}\right]\right)_{k}$ be a sequence in $X(\Gamma, \mathrm{G})$ converging to a point $[\mathrm{L}]$ of $\Omega(\Gamma, \mathrm{G})$. Let $\mu_{\mathrm{k}} \in \mathcal{C}(S)$ be the current with

$$
i\left(\mu_{k}, c\right)=\left\|\lambda\left(\rho_{k}(\gamma)\right)\right\|,
$$

where $\gamma \in \Gamma$ is any hyperbolic element with corresponding closed geodesic $c \subset S$. By [MZ19, Corollary 1.5], we have that

$$
h\left(\rho_{k}\right) \operatorname{Syst}\left(\mu_{k}\right) \leqslant C,
$$

where $C$ is a constant only depending on $S$. We will now show that

$$
\lim _{k \rightarrow \infty} \operatorname{Syst}\left(\mu_{k}\right)=\infty,
$$

which will imply the corollary. Let $b$ be the geodesic current associated to a binding multicurve in $S$. Then, by compactness of $\{\mu \in \mathcal{C}(S): i(\mu, b)=$ $1\}$, up to extracting we have that $\frac{1}{i\left(\mu_{k}, b\right)} \mu_{k}$ converges to some non zero current $\mu$. Then by continuity of $i$ we have

$$
\lim _{k \rightarrow \infty} \frac{1}{i\left(\mu_{k}, b\right)}\left\|\lambda\left(\rho_{k}(\gamma)\right)\right\|=i(\mu, c)
$$

for all hyperbolic $\gamma \in \Gamma$ with corresponding closed geodesic $c \subset S$. As $\mu \neq 0$ this implies that $\lim _{k \rightarrow \infty} i\left(\mu_{k}, b\right)=+\infty$ and $\|L(\gamma)\|=i(\mu, c)$ for all $\gamma$ for some representant L of $[\mathrm{L}]$. In particular we have Syst $(\mu)=\operatorname{Syst}(\mathrm{L})$. By continuity of the systole (Corollary 1.5(1)) we have that

$$
\lim _{k \rightarrow \infty} \frac{1}{i\left(\mu_{k}, b\right)} \operatorname{Syst}\left(\mu_{k}\right)=\operatorname{Syst}(\mu)
$$

As $\operatorname{Syst}(\mu)=\operatorname{Syst}(L)>0$ and $\lim _{k \rightarrow \infty} i\left(\mu_{k}, b\right)=+\infty$, this implies that $\lim _{k \rightarrow \infty} \operatorname{Syst}\left(\mu_{k}\right)=+\infty$.

Turning to Corollary 1.14, we define now the Jordan projection

$$
\lambda: \operatorname{SL}(\mathrm{n}, \mathbb{K}) \rightarrow \mathfrak{C}
$$

for $\mathbb{K}=\mathbb{R}\left[\left[X^{-1}\right]\right]$. Let

$$
\overline{\mathbb{K}}:=\bigcup_{\substack{\mathrm{q} \geqslant 1 \\ \mathrm{q} \in \mathbb{N}}} \mathbb{C}\left[\left[X^{-1 / q}\right]\right]
$$

be an algebraic closure of $\mathbb{K}$. The valuation $v$ defined on $\mathbb{K}$ extends uniquely to $\overline{\mathbb{K}}$ by

if

$$
v(\lambda)=-\frac{\ell}{q}
$$

$$
\lambda=\sum_{\substack{k \leq \ell \\ k \in \mathbb{Z}}} a_{k} X^{k / q}
$$

for $a_{\ell} \neq 0$. Given $g \in \operatorname{SL}(n, \mathbb{K})$, we order the eigenvalues $a_{1}, \ldots, a_{n}$ of $g$ so that

$$
\lambda(g):=\left(-v\left(a_{1}\right), \ldots,-v\left(a_{n}\right)\right) \in \mathfrak{C} .
$$

Let $\|\cdot\|_{2}$ be the Euclidean norm restricted to $\mathfrak{C}$. We have: 
Lemma 7.3. Let $\ell_{\mathcal{B}_{\mathfrak{n}}(\mathbb{K})}(\mathrm{g})$ denote the translation length of $\mathrm{g} \in \mathrm{SL}(\mathrm{n}, \mathbb{K})$ computed with respect to the $C A T(0)$-metric in $\mathcal{B}_{\mathrm{n}}(\mathbb{K})$. Then

$$
\ell_{\mathcal{B}_{\mathfrak{n}}(\mathbb{K})}(\mathrm{g})=\|\lambda(\mathrm{g})\|_{2} .
$$

Proof. Let $\mathbb{K} \subset \mathbb{L} \subset \overline{\mathbb{K}}$ be the splitting field in $\overline{\mathbb{K}}$ of the characteristic polynomial of $\mathrm{g}$. Since $\mathbb{K}$ is complete with discrete valuation and $\mathbb{L}$ is a Galois extension of $\mathbb{K}$, the building $\mathcal{B}_{n}(\mathbb{K})$ embeds $\operatorname{SL}(n, \mathbb{K})$-equivariantly in the building $\mathcal{B}_{n}(\mathbb{L})$ of $S L(n, \mathbb{L})$ as a convex subset, [Tit79, 2.6]. Therefore it suffices to show that

$$
\|\lambda(g)\|_{2}=\ell_{\mathcal{B}_{\mathfrak{n}}(\mathbb{L})}(\mathrm{g}) .
$$

Let $\mathrm{g}=\mathrm{s} u=u s$ be the Jordan decomposition of $\mathrm{g}$ with $s$ diagonalizable in a basis $\mathcal{E}$ of $\mathbb{L}^{n}$ and $u$ unipotent upper triangular in this basis. As $\ell_{\mathcal{B}_{\mathfrak{n}}(\mathbb{L})}(u)=0$, we have that

$$
\ell_{\mathcal{B}_{\mathfrak{n}}(\mathbb{L})}(\mathrm{g})=\ell_{\mathcal{B}_{\mathfrak{n}}(\mathbb{L})}(\mathrm{s}) .
$$

The action by $s$ on the apartment associated to the basis $\mathcal{E}$ in the model of $\mathcal{B}_{n}(\mathbb{L})$ of good norms on $\mathbb{L}^{n}$ (see [Par00, 3.2.2]) is given by translation by $\lambda(\mathrm{s})=\lambda(\mathrm{g})$, which completes the proof of the lemma.

Proof of Corollary 1.14 Let

$$
\overline{\mathbb{K}}^{\mathrm{r}}=\bigcup_{\ell \geqslant 1} \mathbb{R}\left[\left[\mathrm{X}^{-1 / \ell}\right]\right],
$$

endowed with the order

$$
\sum_{k \leqslant \ell} a_{k} X^{k / q}>0
$$

if $a_{\ell}>0$. This order is compatible with the valuation $v$ and $\overline{\mathbb{K}}^{r}$ is real closed. Observe that $\overline{\mathbb{K}}=\overline{\mathbb{K}}^{r}(\sqrt{-1})$ : thus, if $\lambda \in \overline{\mathbb{K}}$, then $\lambda \bar{\lambda} \in \overline{\mathbb{K}}^{\mathrm{r}}$ is positive and we denote

$$
\|\lambda\|=\sqrt{\lambda \bar{\lambda}}
$$

Let $\overline{\mathbb{R}(X)}^{\mathrm{r}}$ be the real closure of $\mathbb{R}(X)$ in $\overline{\mathbb{K}}^{\mathrm{r}}$. Given $g \in \operatorname{SL}(\mathrm{n}, \mathbb{R}(X))$, its eigenvalues $\lambda_{1}, \ldots, \lambda_{n}$ lie in $\overline{\mathbb{R}(X)}^{\mathrm{r}}(\sqrt{-1})$ and we order them so that

$$
\lambda(g)=\left(-v\left(\left|a_{1}\right|\right), \ldots,-v\left(\left|a_{n}\right|\right)\right) \in \mathfrak{C} .
$$

Observe that any $\lambda \in \overline{\mathbb{R}(X)}^{\text {r }}$ can be represented by a Puiseux series that is convergent at $\infty$. As a result, if $\lambda>0$

$$
-v(\lambda)=\lim _{t \rightarrow \infty} \frac{\ln \lambda(t)}{\ln t} .
$$

This implies that for every $\gamma \in \Gamma$

$$
\lim _{t \rightarrow \infty} \frac{\lambda\left(\rho_{t}(\gamma)\right)}{\ln t}=\lambda(\rho(\gamma)) .
$$


Since $\operatorname{tr}\left(\rho\left(\gamma_{0}\right)\right)$ has a pole at infinity, we must have $\lambda\left(\rho\left(\gamma_{0}\right)\right) \neq 0$, from which it follows that $[\lambda \circ \rho] \in \partial X(\Delta, \operatorname{SL}(n, \mathbb{R}))$. Since the latter coincides with $\Omega(\Delta, \operatorname{SL}(n, \mathbb{R}))$ by Theorem 1.13 , this shows (1).

For the second assertion, let $\Gamma<\Delta$ be a torsion-free finite index subgroup. By Lemma 7.3 we have that for all $\gamma \in \Gamma$

$$
\|\lambda(\rho(\gamma))\|_{2}=\ell_{\mathcal{B}_{n}(\mathbb{K})}(\rho(\gamma)),
$$

and Theorem 1.13 and Corollary 1.10 (2) imply then that for some constants $0<\mathrm{c}_{1} \leqslant \mathrm{c}_{2}$,

for all $\gamma \in \Gamma$.

$$
c_{1} \ell_{\text {hyp }}(\gamma) \leqslant \ell_{\mathcal{B}_{\mathfrak{n}}(\mathbb{K})}(\rho(\gamma)) \leqslant c_{2} \ell_{\text {hyp }}(\gamma)
$$

This says that the $\Gamma$-action on $\mathcal{B}_{\mathfrak{n}}(\mathbb{K})$ is displacing and hence [DGLM11, Proposition 2.2.2 and Lemma 2.8.1] imply that the $\Gamma$-orbits, and hence the $\Delta$-orbits, are quasi-isometric embeddings.

\section{REFERENCES}

[ALS18] D. Alessandrini, G.-S. Lee, and F. Schaffhauser. Hitchin components for orbifolds. ArXiv e-prints, November 2018.

[BCLS18] M. Bridgeman, R. Canary, F. Labourie, and A. Sambarino. Simple root flows for Hitchin representations. Geom. Dedicata, 192:57-86, 2018.

[BIPP20] Marc Burger, Alessandra Iozzi, Anne Parreau, and Marie Beatrice Pozzetti. The real spectrum compactification of character varieties: characterizations and applications, 2020. To appear C. R. Math. Acad. Sci. Paris .

[BIPP21] Marc Burger, Alessandra Iozzi, Anne Parreau, and Maria Beatrice Pozzetti. Positive crossratios, barycenters, trees and applications to maximal representations, 2021. https://arxiv.org/abs/2103.17161.

[BIW10] M. Burger, A. Iozzi, and A. Wienhard. Surface group representations with maximal Toledo invariant. Ann. of Math. (2), 172(1):517-566, 2010.

[BIW14] M. Burger, A. Iozzi, and A. Wienhard. Higher Teichmüller spaces: from $\operatorname{SL}(2, \mathbb{R})$ to other Lie groups. In Handbook of Teichmüller theory. Vol. IV, volume 19 of IRMA Lect. Math. Theor. Phys., pages 539-618. Eur. Math. Soc., Zürich, 2014.

[Bon86] F. Bonahon. Bouts des variétés hyperboliques de dimension 3. Ann. of Math. (2), 124(1):71-158, 1986.

[Bon88] F. Bonahon. The geometry of Teichmüller space via geodesic currents. Invent. Math., 92(1):139-162, 1988.

[Bon01] F. Bonahon. Geodesic laminations on surfaces. In Laminations and foliations in dynamics, geometry and topology (Stony Brook, NY, 1998), volume 269 of Contemp. Math., pages 1-37. Amer. Math. Soc., Providence, RI, 2001.

[BP17] M. Burger and M.B. Pozzetti. Maximal representations, non-Archimedean Siegel spaces, and buildings. Geom. Topol., 21(6):3539-3599, 2017.

[CEG06] R. D. Canary, D. B. A. Epstein, and P. L. Green. Notes on notes of Thurston [mr0903850]. In Fundamentals of hyperbolic geometry: selected expositions, volume 328 of London Math. Soc. Lecture Note Ser., pages 1-115. Cambridge Univ. Press, Cambridge, 2006. With a new foreword by Canary.

[DGLM11] Th. Delzant, O. Guichard, F. Labourie, and S. Mozes. Displacing representations and orbit maps. In Geometry, rigidity, and group actions, Chicago Lectures in Math., pages 494-514. Univ. Chicago Press, Chicago, IL, 2011.

[DLR10] M. Duchin, Ch.J. Leininger, and K. Rafi. Length spectra and degeneration of flat metrics. Invent. Math., 182(2):231-277, 2010. 
[Ebe96] P.B. Eberlein. Geometry of nompositively curved manifolds. Chicago Lectures in Mathematics. University of Chicago Press, Chicago, IL, 1996.

[EM] V. Erlandsson and G. Mondello. Ergodic invariant measures on the space of geodesic currents. https://arxiv.org/abs/1807.02144.

[Glo] O. Glorieux. Critical exponent for geodesic currents. https://arxiv.org/abs/1704.06541.

[GM91] F. P. Gardiner and H. Masur. Extremal length geometry of Teichmüller space. Complex Variables, Theory Appl., 16(2-3):209-237, 1991.

[Gol88] W.M. Goldman. Geometric structures on manifolds and varieties of representations. In Geometry of group representations (Boulder, CO, 1987), volume 74 of Contemp. Math., pages 169-198. Amer. Math. Soc., Providence, RI, 1988.

[Lab06] F. Labourie. Anosov flows, surface groups and curves in projective space. Invent. Math., 165(1):51-114, 2006.

[LRT11] D. Long, A. Reid, and M. Thistlethwaite. Zariski dense surface subgroups in SL(3,Z). Geom. Topol., 15(1):1-9, 2011.

[Mar] B. Martelli. An introduction to geometric topology. https:/ /arxiv.org/abs/1610.02592.

[MS91] J.W. Morgan and P.B. Shalen. Free actions of surface groups on R-trees. Topology, 30(2):143-154, 1991.

[MZ19] Giuseppe Martone and Tengren Zhang. Positively ratioed representations. Comment. Math. Helv., 94(2):273-345, 2019.

[Nie15] X. Nie. Entropy degeneration of convex projective surfaces. Conform. Geom. Dyn., 19:318-322, 2015.

[Ota90] J.-P. Otal. Le spectre marqué des longueurs des surfaces à courbure négative. Ann. of Math. (2), 131(1):151-162, 1990.

[Par00] A. Parreau. Immeubles affines: construction par les normes et étude des isométries. In Crystallographic groups and their generalizations (Kortrijk, 1999), volume 262 of Contemp. Math., pages 263-302. Amer. Math. Soc., Providence, RI, 2000.

[Par12] A. Parreau. Compactification d'espaces de représentations de groupes de type fini. Math. Z., 272(1-2):51-86, 2012.

[Tit79] J. Tits. Reductive groups over local fields. In Automorphic forms, representations and L-functions (Proc. Sympos. Pure Math., Oregon State Univ., Corvallis, Ore., 1977), Part 1, Proc. Sympos. Pure Math., XXXIII, pages 29-69. Amer. Math. Soc., Providence, R.I., 1979.

[Zha15a] T. Zhang. The degeneration of convex $\mathbb{R}^{2}$ structures on surfaces. Proc. Lond. Math. Soc. (3), 111(5):967-1012, 2015.

[Zha15b] T. Zhang. Degeneration of Hitchin representations along internal sequences. Geom. Funct. Anal., 25(5):1588-1645, 2015.

Department Mathematik, ETH Zentrum, Rämistrasse 101, CH-8092 ZüRICH, SwitzerLAND

Email address: burger@math.ethz.ch

Department Mathematik, ETH Zentrum, RÄMistrasse 101, CH-8092 ZüRICH, SwitzerLAND

Email address: iozzi@math.ethz.ch

Univ. Grenoble Alpes, CNRS, Institut Fourier, F-38000 Grenoble, France

Email address: Anne.Parreau@univ-grenoble-alpes.fr

Mathematical Institute, Heidelberg University, Im Neuenheimer feld 205, 69120

HEIDELbERG, GERMANY

Email address: pozzetti@mathi.uni-heidelberg.de 